University of Nebraska - Lincoln

DigitalCommons@University of Nebraska - Lincoln

$5-31-2019$

\title{
Opportunities for behavioral rescue under rapid environmental change
}

\author{
Samuel B. Fey \\ Reed College, Feys@reed.edu \\ David A. Vasseur \\ Yale University, david.vasseur@yale.edu \\ Karla Alujević \\ Stellenbosch University, kalujevic@sun.ac.za \\ Kristy J. Kroeker \\ University of California Santa Cruz, kkroeker@ucsc.edu \\ Michael L. Logan \\ University of Nevada, Reno, michaellogan@unr.edu
}

See next page for additional authors

Follow this and additional works at: https://digitalcommons.unl.edu/bioscifacpub

Part of the Biology Commons

Fey, Samuel B.; Vasseur, David A.; Alujević, Karla; Kroeker, Kristy J.; Logan, Michael L.; O'Connor, Mary I.; Rudolf, Volker H.W.; DeLong, John; Peacor, Scott; Selden, Rebecca L.; Sih, A; and Clusella-Trullas, Susana, "Opportunities for behavioral rescue under rapid environmental change" (2019). Faculty Publications in the Biological Sciences. 829.

https://digitalcommons.unl.edu/bioscifacpub/829

This Article is brought to you for free and open access by the Papers in the Biological Sciences at DigitalCommons@University of Nebraska - Lincoln. It has been accepted for inclusion in Faculty Publications in the Biological Sciences by an authorized administrator of DigitalCommons@University of Nebraska - Lincoln. 


\section{Authors}

Samuel B. Fey, David A. Vasseur, Karla Alujević, Kristy J. Kroeker, Michael L. Logan, Mary I. O'Connor, Volker H.W. Rudolf, John DeLong, Scott Peacor, Rebecca L. Selden, A Sih, and Susana Clusella-Trullas 


\title{
Opportunities for behavioral rescue under rapid environmental change
}

\author{
Samuel B. Fey, ${ }^{1}$ David A. Vasseur, ${ }^{2}$ Karla Alujević, ${ }^{3}$ \\ Kristy J. Kroeker, ${ }^{4}$ Michael L. Logan,, ${ }^{5,6}$ Mary I. O'Connor, ${ }^{7}$ \\ Volker H. W. Rudolf, ${ }^{8}$ John P. DeLong, ${ }^{9}$ Scott Peacor, ${ }^{10}$ \\ Rebecca L. Selden, ${ }^{11}$ Andy Sih, ${ }^{12}$ and Susana Clusella-Trullas ${ }^{3}$
}

1 Department of Biology, Reed College, Portland, Oregon

2 Department of Ecology and Evolutionary Biology, Yale University, New Haven, Connecticut

3 Department of Botany and Zoology \& Centre for Invasion Biology, Stellenbosch University, Stellenbosch, South Africa

4 Department of Ecology and Evolutionary Biology, University of California Santa Cruz, Santa Cruz, California

5 Department of Biology, University of Nevada, Reno, Nevada

6 Smithsonian Tropical Research Institute, Panama City, Panama

7 Department of Zoology and Biodiversity Research Centre, University of British Columbia, Vancouver, BC, Canada

8 BioSciences, Rice University, Houston, Texas

9 School of Biological Sciences, University of Nebraska, Lincoln, Nebraska

10 Department of Fisheries and Wildlife, Michigan State University, East Lansing, Michigan

11 Department of Ecology, Evolution, and Natural Resources, Rutgers University, New Brunswick, New Jersey

12 Department of Environmental Science \& Policy, University of California Davis, Davis, California

Samuel B. Fey and David A. Vasseur are first authors and contributed equally to the manuscript.

Correspondence Samuel B. Fey, Department of Biology, Reed College, Portland, OR 97202; email Feys@reed.edu

Published as: Fey SB, Vasseur DA, Alujević K, et al. Opportunities for behavioral rescue under rapid environmental change. Global Change Biology, Volume 25, Issue 9 (September 2019), pp 3110-3120.

doi:10.1111/gcb.14712

Copyright (C) 2019 John Wiley \& Sons Ltd. Used by permission.

Submitted 5 March 2019; accepted 9 May 2019; published 31 May 2019. 
ORCID

Samuel B. Fey https://orcid.org/0000-0002-7471-3308

Kristy J. Kroeker https://orcid.org/0000-0002-5766-1999

Volker H. W. Rudolf https://orcid.org/0000-0002-9214-2000

John P. DeLong https://orcid.org/0000-0003-0558-8213

\begin{abstract}
Laboratory measurements of physiological and demographic tolerances are important in understanding the impact of climate change on species diversity; however, it has been recognized that forecasts based solely on these laboratory estimates overestimate risk by omitting the capacity for species to utilize microclimatic variation via behavioral adjustments in activity patterns or habitat choice. The complex, and often context-dependent nature, of microclimate utilization has been an impediment to the advancement of general predictive models. Here, we overcome this impediment and estimate the potential impact of warming on the fitness of ectotherms using a benefit/ cost trade-off derived from the simple and broadly documented thermal performance curve and a generalized cost function. Our framework reveals that, for certain environments, the cost of behavioral thermoregulation can be reduced as warming occurs, enabling behavioral buffering (e.g., the capacity for behavior to ameliorate detrimental impacts) and "behavioral rescue" from extinction in extreme cases. By applying our framework to operative temperature and physiological data collected at an extremely fine spatial scale in an African lizard, we show that new behavioral opportunities may emerge. Finally, we explore large-scale geographic differences in the impact of behavior on climate-impact projections using a global dataset of 38 insect species. These multiple lines of inference indicate that understanding the existing relationship between thermal characteristics (e.g., spatial configuration, spatial heterogeneity, and modal temperature) is essential for improving estimates of extinction risk.
\end{abstract}

Keywords behavioral thermoregulation, climate change, ecological forecasting, environmental variation, macrophysiology, temperature

\title{
1 Introduction
}

A critical challenge in current ecological research is to understand how environmental temperature regimes limit the abundance and distribution of species with sufficient accuracy to enable predictions of the future effects of climate change (Kearney, Shine, \& Porter, 2009; Porter \& Kearney, 2009; Williams, Shoo, Isaac, Hoffmann, \& Langham, 2008). Recent advances have emphasized organismal traits, such as the thermal dependence of population growth rate, as the mechanistic basis for understanding how changing environmental conditions may determine the vulnerability of populations (particularly ectotherm populations) to extinction (Dell, Pawar, \& Savage, 2013; Kearney \& Porter, 2009; Kearney et al., 2009; Litchman \& Klausmeier, 2008; Sinervo et al., 2010). 
However, while thermal performance traits are usually assessed in controlled laboratory conditions, performance in nature is mediated by the ability of ectothermic organisms to manage their body temperature behaviorally (Buckley, Ehrenberger, \& Angilletta, 2015; Long et al., 2014; Porter \& Gates, 1969). In addition to the physiological mechanisms used to heat and cool their bodies (Holland, Brill, Chang, Sibert, \& Fournier, 1992), ectotherms regulate their body temperature through microhabitat choice (Huey, 1974; Logan, Huynh, Precious, \& Calsbeek, 2013; Porter, Mitchell, Beckman, \& DeWitt, 1973; Scheffers, Edwards, Diesmos, Williams, \& Evans, 2014; Scheffers, Evans, Williams, \& Edwards, 2014; Woods, Dillon, \& Pincebourde, 2015), thereby expending energy to seek out areas within their habitat that are thermally favorable (e.g., behaviorally thermoregulating; Kearney et al., 2009; Sears et al., 2016; Sunday et al., 2016). The decision to thermoregulate or thermoconform has been shown to depend on the cost of thermoregulation (Huey, 1974; Huey \& Slatkin, 1976); these costs are strongly dependent on the spatial structure of the thermal environment (Huang, Porter, Ming-Chung, \& Chiou, 2014; Pincebourde, Murdock, Vickers, \& Sears, 2016; Sears et al., 2016) and have been refined by recent advances in the resolution of spatial data at the small and microscales important for many ectotherms (Logan et al., 2013; Scheffers, Edwards, et al., 2014; Scheffers, Evans, et al., 2014; Woods et al., 2015). Therefore, accurate ecological forecasts amidst climate warming necessitate a tractable approach for harmonizing how these categories of environmental and organismal data inform each other.

Despite decades of research highlighting the importance of behavior to mediate fitness, animal behavior has been excluded from many recent broad-scale climate-impact projections (Deutsch et al., 2008; Thomas, Kremer, Klausmeier, \& Litchman, 2012; Vasseur et al., 2014), which generates several potential sources of potential bias. First, assuming movement behavior is adaptive (i.e., that it increases physiological performance) forecasts ignoring behavior will generally overemphasize the detrimental impacts of warming (Pincebourde et al., 2016), particularly when the net benefits of behavioral thermoregulation remain high during periods of elevated environmental temperatures (Sears, Raskin, \& Angilletta, 2011). Indeed, the ability of organisms to behaviorally thermoregulate currently enables the persistence of populations in environments that would otherwise exceed physiological limits (Sunday et al., 
2016), and is predicted to be a key ecological feature enabling the persistence of populations in response to sustained climate warming (Huey et al., 2012; Kearney et al., 2009; Sears et al., 2016). Yet, the anticipated net benefits of thermoregulatory behaviors will vary dramatically across organisms and ecosystems - ranging from situations where environmental warming may provide new opportunities for behavior to increase fitness above current levels (Huey, Hertz, \& Sinervo, 2003; Logan et al., 2013) to instances where, despite movement abilities and the capacity for behavioral thermoregulation, climate warming will likely restrict the ability of organisms to survive and reproduce (Caillon, Suppo, Jérôme Casas, Woods, \& Pincebourde, 2014; Sinervo et al., 2010). As such, understanding how to modify macroecological patterns of climate impact projections to include behavior is of high priority.

The factors influencing decisions surrounding behavioral thermoregulation are myriad (Basson, Levy, Angilletta, \& Clusella-Trullas, 2017; Porter et al., 1973; Sears et al., 2016), but the winning thermoregulatory strategy ultimately depends on the relative costs and benefits associated with movement behavior (Huey, 1974; Huey \& Slatkin, 1976). While much research has focused on how features of organisms (e.g., body size, velocity, reliance on evaporative cooling; Huey et al., 2012; Huey \& Slatkin, 1976; Kearney et al., 2009; Porter \& Kearney, 2009; Shine \& Kearney, 2001; Sunday et al., 2016) or properties of the environment (e.g., the available and configuration of preferred thermal habitat, or the modal environmental temperature) impact the costs of behavioral thermoregulation and ultimately fitness (Huey \& Slatkin, 1976; Logan, Fernandez, \& Calsbeek, 2015; Logan et al., 2013; Martin \& Huey, 2008; Sears et al., 2016, 2011), little emphasis has been placed on understanding how the existing relationship between thermal characteristics may influence the costs associated with behavioral thermoregulation. Yet, these relationships (e.g., the existence of a correlation between spatial heterogeneity and mean temperature) define the conditions over which costs and benefits of thermoregulation will be realized during environmental extremes and ultimately shape the constraints and opportunities for behavior to rescue populations from being locally extirpated amidst changing climatic conditions.

Here, we identify that, and explore how, existing relationships between thermal characteristics can constrain or enable opportunities for behavioral thermoregulation amidst warming. We begin by presenting 
a general framework that incorporates thermal physiology and spatiotemporal variability in environmental temperature to predict thermoregulatory behavior and ultimately the performance of ectotherms in nature. Our approach is not intended to displace the important and necessary work on the physics and physiology of behavioral thermoregulation (e.g., Kearney \& Porter, 2009; Shine \& Kearney, 2001), rather to describe the environmental conditions that are most ripe for behavior to play an important role under climate change and to expand our ability to update the extinction budget for larger classes of organisms. We use this approach to explore how relationships between the spatial mean temperature and the costs of behavioral thermoregulation can influence estimates of fitness. Next, using highly resolved environmental and physiological data from an African lizard, we highlight how warming may provide new opportunities for thermoregulation by altering its underlying cost. Finally, we explore large-scale geographic differences in the predicted impact of behavior on climate-impact projections using a global dataset of 38 insect species and a set of hypotheses about the change in spatial microclimate variation with climate change. Collectively our results indicate that the relationship between the mean temperature, the costs of behavioral thermoregulation, and the structure of microclimatic variation, should be a focal consideration in ongoing climate change research.

\section{Theoretical framework}

Our framework predicts field performance, which we define as the timeaverage realized value of the aggregate of vital physiological processes affecting an individual in a spatiotemporally variable environment. We use the term "field performance" instead of "fitness" because most measures of performance (e.g., survival, swimming speed, eggs produced) do not wholly define the absolute fitness of an individual (Angilletta, 2009; Logan et al., 2013). Field performance can be measured over many individuals in a population to develop a metric of mean population fitness. We develop a cost-benefit model that identifies the body temperature for an individual organism that maximizes performance given its thermal physiology, the current state of the thermal environment, and the costs associated with locomotion to a different microclimate within the 
broader environment. Here, the thermal environment is represented by a multidimensional, spatially explicit thermal landscape, $\mathbb{T}$, that is divided into regular discrete microhabitats on a scale that is biologically relevant to the individual organism and characterized by known probability density and autocorrelation functions (Figure 1a). In the absence of behavioral thermoregulation, we assume that an individual is situated at the modal value in this landscape and that its body temperature $\left(T_{\mathrm{b}}\right)$ mimics that of its current microhabitat, such that $T_{\mathrm{b}}=\overline{\mathbb{T}}$, which is true so long as the distribution of temperatures across the landscape is unimodal without skewness. The performance of this individual across different body temperatures is given by the thermal performance curve, $P\left(T_{\mathrm{b}}\right)$ (Figure 1b,c, thick black curve). This curve is commonly measured under constant laboratory conditions and has well-studied properties (Angilletta, 2006; Deutsch et al., 2008; Thomas et al., 2012). Various mathematical relationships have been proposed to describe $P\left(T_{\mathrm{b}}\right)$ (Angilletta, 2006; Deutsch et al., 2008; Sears \& Angilletta, 2015; Thomas et al., 2017); here, we use the unimodal function

$$
P(T)=b_{1} \exp \left(b_{2} T\right)-\left(d_{0}+d_{1} \exp \left(d_{2} T\right)\right)
$$

where the first and second terms of this function correspond to the effect of temperature on birth and death rates, respectively (Thomas et al., 2017).

The benefit (measured in the currency of field performance) of altering body temperature from the initial condition, $\overline{\mathbb{T}}$, is given by the performance differential (Figure 1b,c, dashed axes with origin $\left(\overline{\mathbb{T}}, P_{\overline{\mathbb{T}}}\right)$ ):

$$
B\left(T_{\mathrm{b}}, \overline{\mathbb{T}}\right)=P\left(T_{\mathrm{b}}\right)-P(\overline{\mathbb{T}})
$$

We assume that benefits arise via locomotion to a more favorable location; however, Equation (1) is general for any form of thermoregulation. The benefit function (Equation 1) implicitly integrates all maintenance costs that are not directly associated with thermoregulation, and, therefore, yields negative values whenever maintenance costs exceed energetic benefits. Huey and Slatkin (1976) suggested that the remaining costs of thermoregulation (those not associated with maintenance; e.g., the energetic costs of locomotion) should be an increasing function of the difference between body temperature and the initial condition 
(a)

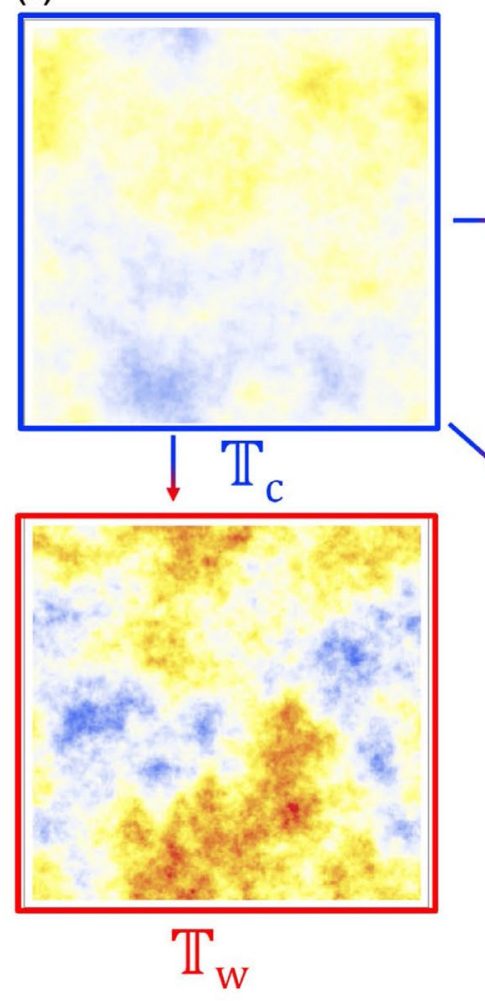

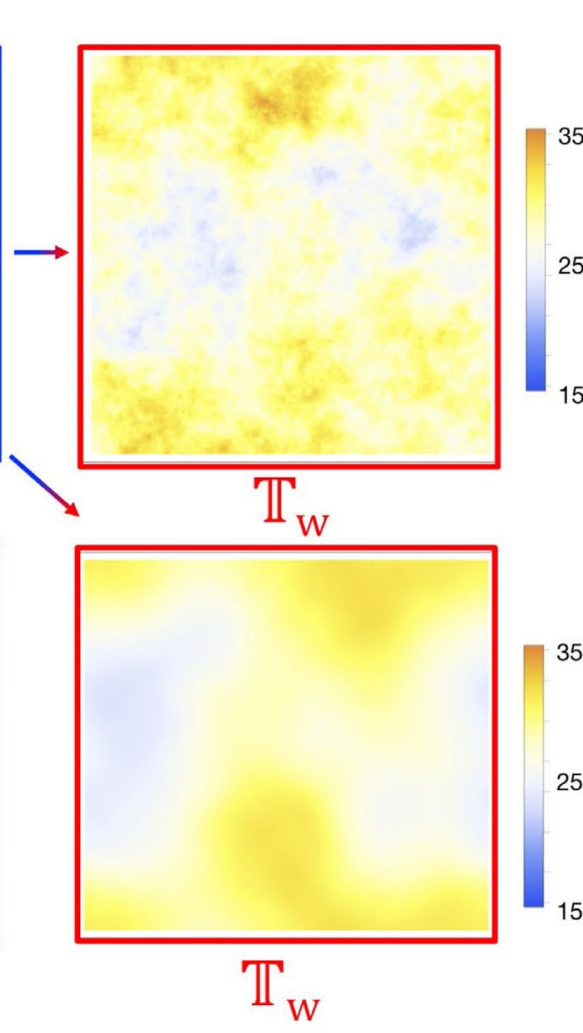

(b)

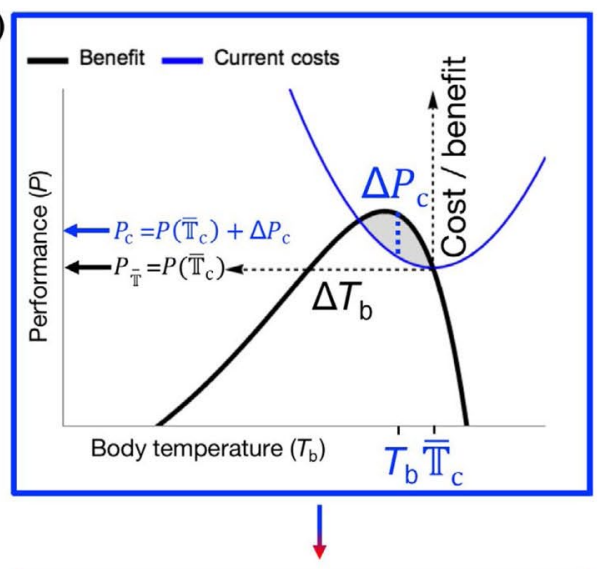

(c)

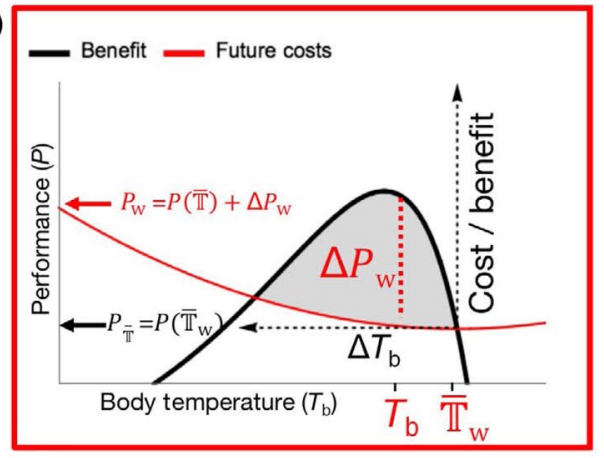

Figure $1 \mathrm{~A}$ framework for estimating the performance consequences of behavioral thermoregulation in warming environments. (a) A thermal environment, blue $\mathbb{T}_{\mathrm{c}^{\prime}}$, represents cool conditions (top left). Warmed environments ( $r e d \mathbb{T}_{w}$ ) have increased mean temperatures, $\overline{\mathbb{T}}$ and relative to cool conditions, are otherwise identical (top right), have increased spatial heterogeneity (bottom left), or have increased spatial autocorrection (bottom right). (b, c) How warming impacts future performance depends on the magnitude of increase of $\overline{\mathbb{T}}$ and the cost of behavioral thermoregulation within $\mathbb{T}_{\mathrm{w}}$. The thermal performance curve (thick black curve) defines the relationship between body temperature, $T_{\mathrm{b}}$, and performance, $P$. For an environment with mean temperature $\overline{\mathbb{T}}$, the benefit, $B$, from altering body temperature from $T_{\mathrm{b}}=\overline{\mathbb{T}}$ is defined by $\Delta P$ relative to $P\left(T_{\mathrm{b}}=\overline{\mathbb{T}}\right)$. The cost of altering body temperature is defined by a cost function $C$ : blue curve in (b) represents the cost function for the cool environment and red curve in (c) represents the cost function for a hypothetical warmed environment that has reduced costs of behavioral thermoregulation. The predicted $P$ and $T_{b}$ for a given $\mathbb{T}$ is calculated as the temperature that maximizes $P$ given $B$ and $C$.

$\left(\Delta T_{\mathrm{b}}=T_{\mathrm{b}}-\overline{\mathbb{T}}\right)$ to reflect the growing costs of maintaining body temperatures that deviate further from the modal temperature of the environment. For behavioral thermoregulation, energetic costs can be influenced by the distribution and spatial configuration of environmental 
temperatures (Huey, 1974; Sears et al., 2016), the morphology, body size, physiology, and search efficiency of the organism, and costs from antagonistic interactions with con- and hetero-specifics (Adolph \& Porter, 1993; Grant \& Porter, 1992). For these reasons, we define a flexible and symmetric cost function as:

$$
C\left(\left|T_{\mathrm{b}}-\overline{\mathbb{T}}\right|\right)=\frac{\beta\left(T_{\mathrm{b}}-\overline{\mathbb{T}}\right)^{2}}{2}
$$

where $\beta$ is a positive-valued parameter that determines the second derivative of the cost function with respect to the absolute temperature deviation $\left|T_{\mathrm{b}}-\overline{\mathbb{T}}\right|$. Large values of $\beta$ generate a steep function to represent a high cost of thermoregulation, whereas low values generate a flatter function to represent a low cost (Figure $1 \mathrm{~b}$ blue line $=$ high $\beta$; Figure $1 \mathrm{c}$ red line $=$ low $\beta$ ). Our simulations of the energetic costs of movement by organisms using different thermoregulatory behaviors and occurring in thermal environments that differ in the extent, autocorrelation, and distribution of thermal spatial heterogeneity (Figures S1-S7, Appendix 1), reveal that Equation (2) can accurately represent the energetic costs associated with locating favorable microhabitats (Figures S1-S7). We assume a linear relationship between the energetic costs of movement and the other environmental and ecological attributes that will influence movement (e.g., threats experienced from predators; Waldschmidt, Jones, \& Porter, 1986). However, ecological factors associated with specific thermal environments (e.g., high resource densities disproportionally occurring in suboptimal thermal environments) may nonlinearly change the shape of the cost function or may result in unanticipated advantages (Fey \& Vasseur, 2016; Rusch \& Angilletta, 2017). Thus, the ways in which multiple, sometimes competing factors impact the measured cost functions remains an important avenue for future research.

Given the benefit and cost functions, the body temperature that maximizes individual performance is that which maximizes the difference between $B\left(T_{\mathrm{b}}, \overline{\mathbb{T}}\right)-C\left(\left|T_{\mathrm{b}}-\overline{\mathbb{T}}\right|\right)\left(T_{\mathrm{b}}\right.$ in Figure $\left.1 \mathrm{~b}, \mathrm{c}\right)$, for example, the conditions that maximize the selective advantage defined by

$$
\left(B\left(T_{\mathrm{b}}, \overline{\mathbb{T}}\right)-B\left(T_{\mathrm{b}}=\overline{\mathbb{T}}, \overline{\mathbb{T}}\right)\right)-\left(C\left(T_{\mathrm{b}}-\overline{\mathbb{T}} \mid\right)-C\left(\left|T_{\mathrm{b}}=\overline{\mathbb{T}}\right|\right)\right)
$$


We refer to this as the predicted body temperature in the field. In the limiting case, when the costs of thermoregulation are extremely low, the performance associated with the predicted body temperature in the field approaches the maximum value defined by the thermal performance curve, regardless of mean environmental temperature. Alternatively, when the costs of thermoregulation are exceedingly high, performance converges on that predicted by $P(\overline{\mathbb{T}})$. In our framework these two limiting cases (perfect thermoregulation and perfect thermoconformity) form the end points on a continuum of potential behavioral strategies (Huey \& Slatkin, 1976).

Changes to the distribution and autocorrelation of $\mathbb{T}$ have different effects on the benefit and cost functions. Alterations to $\mathbb{T}$ generate a translation of the benefit function but do not alter its shape (e.g., shifting $\overline{\mathbb{T}}$ to the optimal value for performance shifts the entire benefit function to values $\leq 0$ ); however, the cost function does not display a simple mechanistic response. In all likelihood, the cost parameter $\beta$ is intricately linked to the thermal landscape. For example, it is likely that $\beta$ scales inversely with the spatial variance of $\mathbb{T}$ if this increases the frequency of desirable conditions in the landscape (Figure S2). Likewise, spatial autocorrelation can greatly alter travel distances between patches of similar and disparate conditions with greater spatial autocorrelation resulting in higher costs of thermoregulatory behavior (Huey, 1974; Sears \& Angilletta, 2015; Figure S1). Without loss of generality, we subsequently examine scenarios where an increase in the mean temperature of the landscape $\mathbb{T}$ is coupled either to a decrease, an increase, or no change in the costs of thermoregulatory behavior.

\section{Materials and methods}

\subsection{Mathematical model and analysis of global insect database}

We used a cost function with a positive second derivative (see Appendix S1, Figures S1-S7) to explore how different cost scenarios impact estimates of performance. Benefit functions for each of these species that describe performance in relation to temperature can be derived from thermal performance curves. For the cost function, however, we have no a priori expectation for how differences among species, 
habitat, geolocation, and life history are manifested. We instead employed a set of nine cost scenarios that arise from crossing three assumptions about the basal cost of thermoregulation $b_{0}=\{0.15,0.5,2.0\}$, with three assumptions about the temperature dependence of such costs $b_{1}=\{-1,0,1\}$ using Equation 2 (Figure S8). Based on the ability of some species to maintain stable body temperatures in spatially heterogeneous environments with high mean temperatures (Sears et al., 2016), the lower end of the costs represent a conservative estimate of the conditions that wild organisms face in nature.

Estimates of performance for a hypothetical organism were determined based on a benefit function defined by

$$
\mathrm{w}\left(T_{\mathrm{b}}\right)=a \times \exp \left(b \times T_{\mathrm{b}}\right)-\left[c+d \times \exp \left(e \times T_{\mathrm{b}}\right)\right]
$$

(Thomas et al., 2017), and by previously described thermal fitness curves for the insect database compiled by Deutsch et al. (2008). For many representations of $\mathrm{w}\left(T_{\mathrm{b}}\right)$, the body temperature that maximizes fitness can be analytically solved as a function of $T_{\mathrm{b}}$; however, the use of a piecewise function for thermal performance (e.g., those in Deutsch et al., 2008) requires numerical maximization. The insect analysis of historic (the decade proceeding when the thermal performance curve was described) and future (2050-2059) temperature distributions were determined according to the previously described methods (Vasseur et al., 2014) based on low-cost (Figures 2c and 4) or multiple-cost environments (Figure S9). We consider a mean performance at or below 0 , equivalent to local extirpation of the population. Computations were performed using Mathematica v. 10.0.

\subsection{Southern rock agama data collection and analysis}

The southern rock agama (Agama atra) is a highly territorial, insectivorous lizard that lives on rock outcrops throughout a large portion of Southern Africa. Males actively defend small territories that are typically composed of several adjacent boulders. We characterized the thermal heterogeneity of three individual lizard territories at Jonaskop $\left(\sim 34^{\circ} \mathrm{S}\right.$, 1,500 m a.s.l.), Western Cape Province, South Africa using 30 physical models in each territory ("operative temperature models" or OTMs; see Appendix S2). Subsequently, we measured the thermal dependence of 

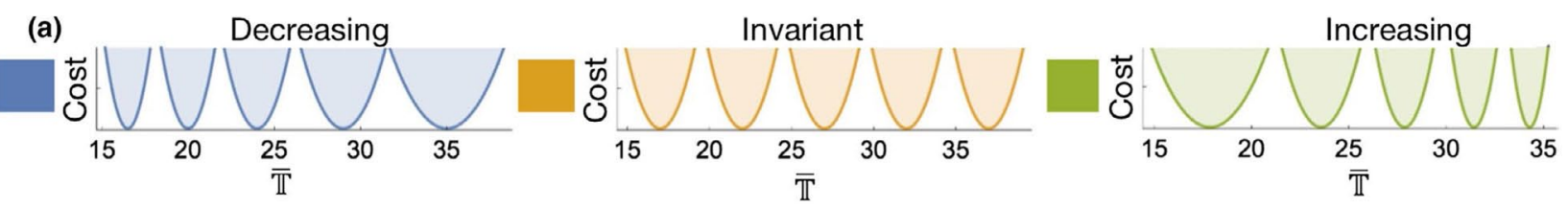

(b)
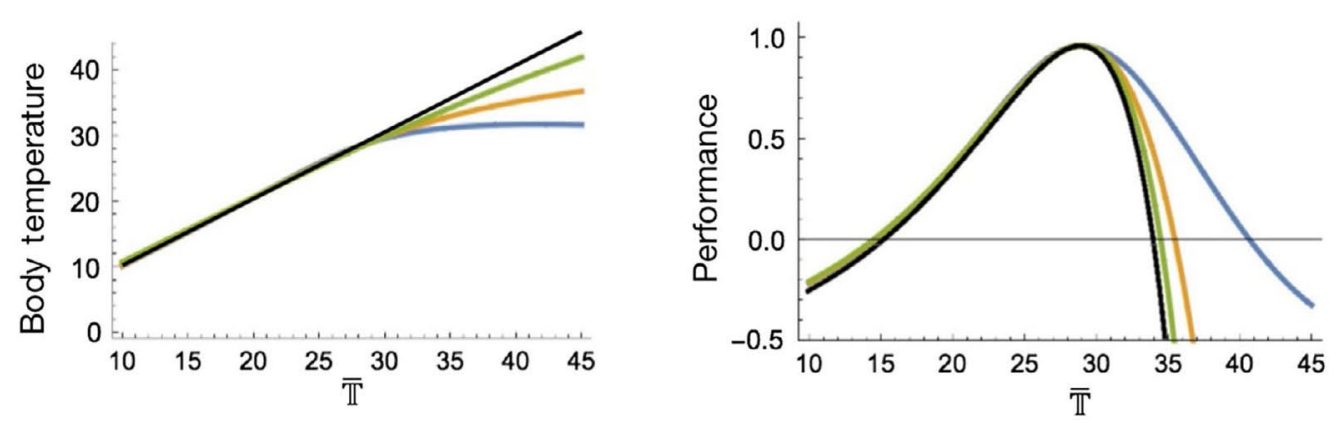

(c)
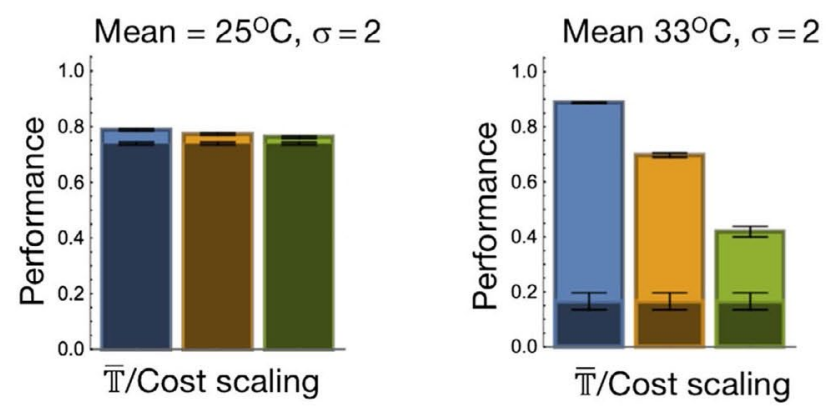

Figure 2 The extent to which thermoregulatory behavior modifies population fitness depends on its cost. (a) Three scenarios for whether the cost of behavioral thermoregulation decreases (blue, A1), is invariant (yellow, A2), or increases (green, A3) with an increase in mean temperature, $\overline{\mathbb{T}}$. Predicted body temperatures (b; left panel) and corresponding performance (b; right panel) for a hypothetical organism based on environments with different relationships between $\overline{\mathbb{T}}$ and cost scaling scenarios; black lines show a scenario where behavior is not allowed. (c) The performance (mean \pm 1 $S E$ ) of a hypothetical ectotherm in a thermal environment with a mean temperature below (left panel, $25^{\circ} \mathrm{C}$ and standard deviation $\sigma=2$ ) and above (right panel, $33^{\circ} \mathrm{C}$ and standard deviation, $\sigma=2$ ) its $T_{\text {opt }}$ of $29^{\circ} \mathrm{C}$; colors correspond to the cost scenarios in (a); light shading indicates the extent to which behavior can increase performance.

the running speed of 37 adult male lizards at the same site across a range of body temperatures: $15,25,30,35,38,42$, and $44^{\circ} \mathrm{C}$ (see detailed methods in Appendix S2, Figure S10, Table S2).

We determined the relationship between the cost of thermoregulation and the spatial mean temperature, as well as between spatial standard deviation and mean temperature using fixed effects linear models. 
To estimate the energetic costs for $A$. atra within a territory, we generated one thermal landscape per lizard territory at each 15 min interval by arranging operative temperature measurements into a 5 by 6 array. To predict operative temperatures in the spaces between loggers, we linearly interpolated the thermal configuration of each territory as a 40 by 40 square array to assemble a total of 2,729 individual landscapes for this temporal window. We utilized previously described methods (see model analysis) to numerically estimate the cost function associated with each landscape as the mean distance needed to reach any possible temperature and predicted performance both with and without behavior at each time point.

To predict the performance of $A$. atra in response to climate warming, we increased $\overline{\mathbb{T}}$ to $+5^{\circ} \mathrm{C}$ above the measured $\overline{\mathbb{T}}$, and evaluated performance with and without behavior for each temperature regime. As such, this scenario assumes that climate warming will occur uniformly and will not affect the existing relationship between environmental temperature and the cost of thermoregulation. We examined the impact of the cost-mean temperature relationship on performance by fitting a linear model between these variables (Figure $3 \mathrm{~b}$ ). We then adjusted this slope to more negative (e.g., a steeper negative relationship between mean temperature and movement cost) and less negative (e.g., values approaching zero and positive numbers) values, while preserving the predicted cost associated with the grand mean of these data.

\subsection{Geographic variation in the importance of behavior}

Using the dataset compiled by Deutsch et al. (2008), we quantified the importance of behavior in historical and future climate regimes for 38 species of insects. We utilize the same methods presented by Vasseur et al. (2014) to determine the average long-term performance for a decade of historic thermal conditions (those most appropriate for the time and location at which the species were collected) and future 2050 scenario (modeled using CGCM3.1/T47), modified to include the behavioral filter described in this paper. We modeled the cost function by setting $\beta=c_{0} e^{c_{1}(T-20)}$, where $c_{0}$ sets the basal cost and $c_{1}$ determines the cost by temperature scaling. We evaluated the dataset using $c_{0}=\{0.15,0.5,2.0\}$ and $c_{1}$ on the interval $(-0.25,0.25)$. The output shown in Figure $4 \mathrm{a}, \mathrm{b}$ assumes a low basal cost $\left(c_{0}=0.15\right)$ and a moderate negative relationship 

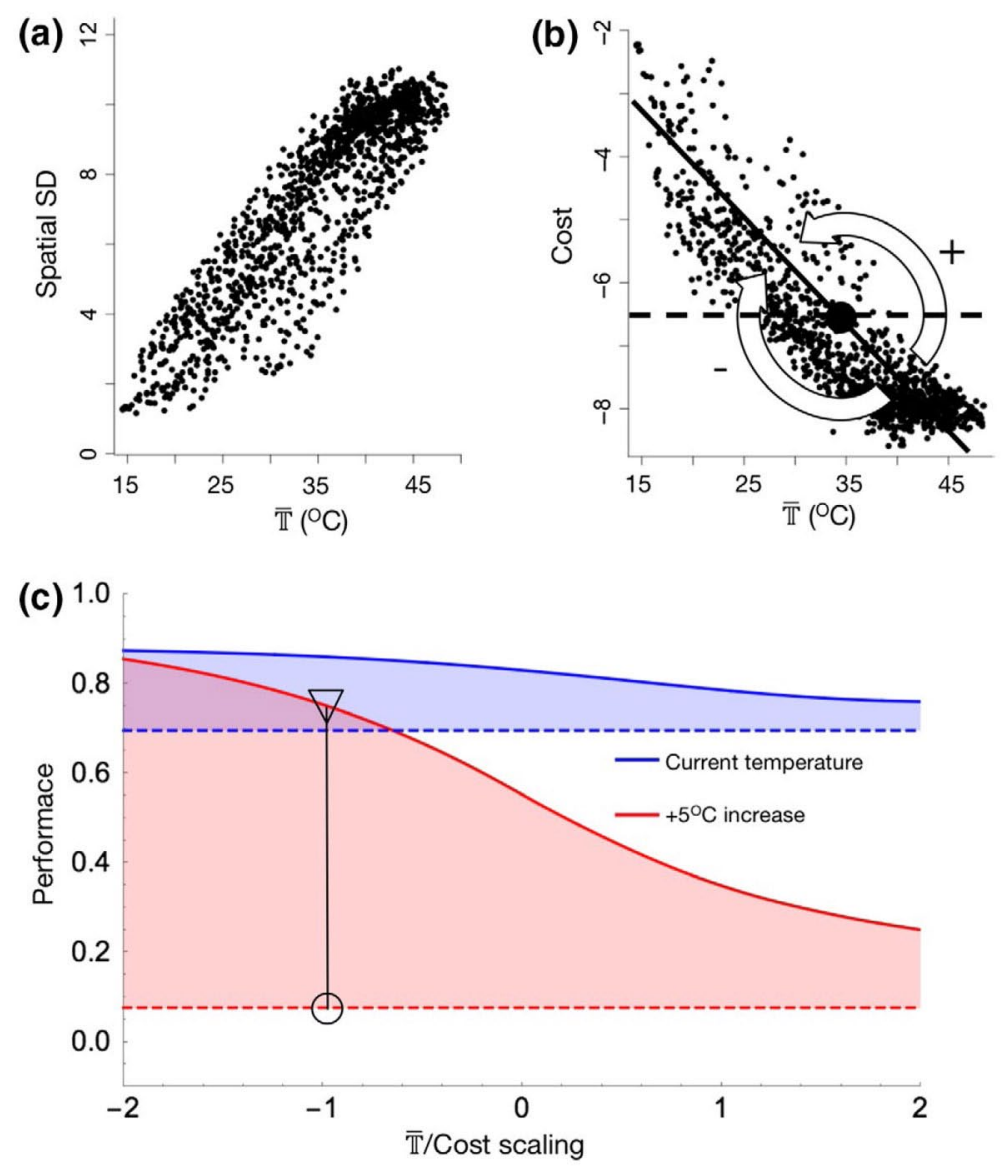

Figure 3 The cost of thermoregulatory behavior influences the predicted performance of southern rock agamas. (a) Spatial standard deviation in environmental temperature increases with mean temperature, $\overline{\mathbb{T}}$, within individual lizard territories; (b) the cost of thermoregulatory behavior ( $C$ in Equation 2 ) decreases as mean temperatures increase. The dashed black line indicates no relationship between $\overline{\mathbb{T}}$ and cost. (c) Increasing the existing cost- mean temperature slope (plus sign in panel b) decreases predicted Agama atra performance, especially in warmer environments. Dashed lines represent performance estimates without behavior in current (blue) and warmed (red) environments; solid lines represent performance estimates with behavior with different cost-mean temperature slope relationships. The triangle and circle indicate performance estimates with and without behavior given the existing $\overline{\mathbb{T}}$ /cost scaling (normalized to -1), respectively. 0 indicates no relationship between cost and mean temperature (dashed line in b), and positive values indicate a positive relationship between cost and mean temperature; $\overline{\mathbb{T}}$ are equivalent in all instances.

between cost and temperature $\left(c_{1}=-0.1\right)$. Other values of these parameters give qualitatively similar patterns but alter the extent to which behavior impacts performance (see Figure S9). 

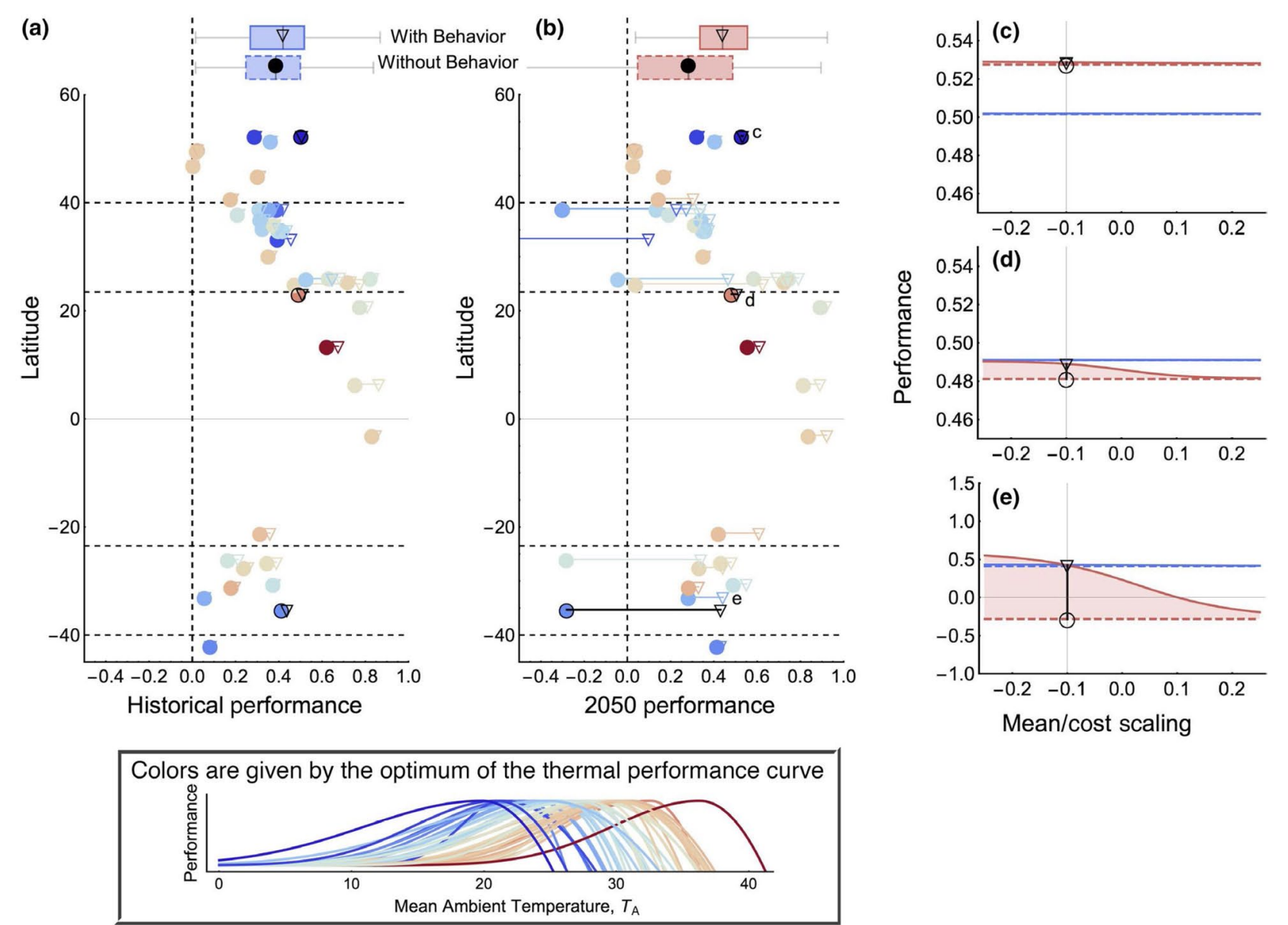

Figure 4 Constraints and opportunities for behavioral rescue vary with latitude. Estimated historic (a) and future (b) performance of 38 insect species assuming no behavior (closed circles) and behavior (open triangles) across latitudes (dash lines depict subtropical latitudes between $\sim 23$ and $40^{\circ}$ ). The inset (box plot) describes the mean performance of all species with (solid) and without (dashed boxes) thermoregulatory behavior. (c-e) Performance as a function of the relationship between the cost of thermoregulation and mean temperature, $\overline{\mathbb{T}}$, in temperate (c), subtropical (d), and tropical (e) regions. Dashed lines represent performance estimates without behavior in historic (dashed blue) and 2050 (dashed red) environments; solid lines represent performance estimates allowing behavior in historic (solid blue) and 2050 (solid red) environments. Negative $\mathrm{x}$-axis values indicate a negative relationship between mean temperature and the cost of thermoregulation; vertical lines are the cost-structure values used in panels a and b. See Figure $S 9$ for a detailed list of performance versus cost scaling relationships for all species. 


\section{Results}

\subsection{Evaluating opportunities for behavioral rescue}

Our model predicts that behavior exerts the greatest effect on field performance when average movement costs are low (e.g., when available thermal heterogeneity is high and spatial autocorrelation is low; Figures S1 and S2) or when the cost of thermoregulatory behaviors decrease with increasing spatial mean temperature (Figure 2 blue), which is consistent with previous findings (Huey, 1974; Sears et al., 2011). In these instances, the accelerating rate at which the benefits of thermoregulation accrue $\left(\mathrm{d} B^{2} / \mathrm{d} T_{\mathrm{b}}^{2}\right)$ relative to the rate at which costs increase $\left(\mathrm{d} C^{2} / \mathrm{d} T_{\mathrm{b}}^{2}\right)$ are conducive to thermoregulatory behavior (Figure $2 \mathrm{a}$ ) and this behavior ultimately elevates performance (Figure 2b,c). Driven by the shape of the thermal performance curve, the benefits of thermoregulation accrue most rapidly when individuals inhabit an environment with a $\overline{\mathbb{T}}$ above which maximal performance is achieved, $T_{\text {opt }}$ (Figure 2b, Figure 2c left vs. right panel), also noted by Martin and Huey (2008). Conversely, in environments with temperatures lower than the $T_{\text {opt }}$, our model predicts lesser change in performance from incorporating behavior because benefits of behavior accrue slowly (Figure 2b,c), supporting the general observation that thermoregulatory behavior is used by many ectotherms to decrease body temperature (Grant \& Dunham, 1988; Kearney et al., 2009; Logan et al., 2015; Sunday et al., 2016). Yet, we do not negate the importance of behavioral thermoregulation strategies that increase body temperature above $\overline{\mathbb{T}}$ (e.g., basking; Huey et al., 2003). Thus, behavioral thermoregulation becomes critically important as climate warming pushes environmental temperatures above $T_{\mathrm{opt}}$ and effective behavioral buffering in these potentially novel conditions requires the availability of thermally suitable microsites.

\subsection{The potential for behavioral rescue in an African lizard}

We apply our framework using data collected in the laboratory and field for an African lizard, the southern rock agama (A. atra). We collected high-resolution spatiotemporal operative temperature data at the scale of individual lizard territories (Figures S11 and S12) and characterized the thermal performance curves for lizards within this population (see 
Materials and Methods) to investigate (a) the extent to which behavioral buffering can ameliorate the detrimental impacts of warming, and (b) the relationship between increasing mean temperature and the likelihood of behavioral rescue (e.g., Figures $2 \mathrm{a}$ and $4 \mathrm{c}-\mathrm{e}$ ). We observed a positive linear relationship between mean temperature and the spatial standard deviation of operative temperatures within territories (Figure 3a). The slope of this relationship remained positive for all active hours (Figure S13), and after restricting the maximum temperature microhabitat the lizards could inhabit (Figure S14). Consistent with our hypothesis, the cost of thermoregulation as measured by $c$ (Equation 2) decreased as $\overline{\mathbb{T}}$ increased (Figure $3 b$, Figure S15).

Forecasts of $A$. atra performance that include movement behavior differ substantially from those that do not include behavior (Figure 3c). Given the observed negative relationship between the cost of thermoregulation and warming in $A$. atra territories, our framework predicts that warming will provide a slight increase in agama performance when movement behavior is allowed (Figure 3c, triangle) relative to estimates of current performance that exclude behavior. By contrast, we anticipate a $\sim 90 \%$ decrease in performance when behavior is not allowed (Figure 3c, circle). To determine the importance of the link between warming and thermoregulatory costs, we varied the strength and sign of this relationship in a simulated landscape. In thermal environments with identical $\overline{\mathbb{T}}$, but with different relationships between thermoregulatory cost and mean temperature, the potential for behavioral buffering can be substantially reduced and performance markedly declines with warming (Figure 3c, red solid line). Thus, the behavioral buffering predicted for these lizards occurs because the hottest time periods coincide with the greatest spatial variation in environmental temperature, thereby reducing the cost of thermoregulatory behavior at the point when behavior is most critical in maintaining physiologically optimal body temperatures. A. atra are, therefore, predicted to be able to maintain sufficiently low body temperatures during hot periods and their body temperatures rarely exceed their critical thermal maximum.

\subsection{Geographic variation in the importance of behavior under climate change}

We applied our framework to population growth rate estimates of 38 insect species from around the globe (Deutsch et al., 2008), assuming the 
same cost structure applies to all species. We evaluated the potential for behavior to alter vulnerability to climate change by explicitly considering how the net benefits of thermoregulatory behavior change across latitude. By estimating the performance of each population in historic and future climates, while either allowing or not allowing behavior (see Materials and Methods), we determine the conditions that favor a large impact of behavior on performance.

In the absence of thermoregulatory behavior, future insect performance decreased by $14 \%$ on average relative to historic performance based on projected local environmental temperatures for 2050 (see Materials and Methods). Irrespective of the assumptions made about the cost of thermoregulation, movement behavior was estimated to have a negligible impact on historical estimates of performance (Figure 4a). By contrast, thermoregulatory behavior has an increased and potentially critical role in future environments because $\overline{\mathbb{T}}$ increasingly rises above $T_{\text {opt }}$ for extended periods of time (Figure $4 \mathrm{~b}$ ).

The inclusion of thermoregulatory behavior in models that predict the effects of thermal regimes on performance can lead to predictions of increased performance, but the extent of this improvement is largely contingent upon the costs of behavior and how such costs change with mean temperature (Figure 2c; Figure S8). The true structure of the cost function remains largely unresolved. Such "behavioral buffering" can alleviate much of the detrimental impacts of warming if the cost of thermoregulatory behavior lessens as mean temperature increases. For example, of the 19 insect species we estimate to be negatively impacted by climate warming, our model predicts that behavior will help alleviate this impact for 17 species, including six instances of "behavioral rescue" whereby behavior saves a population that would otherwise be rendered inviable (estimated performance $<0$ ) by climate change. Furthermore, for 10 species, the inclusion of behavior causes the effects of warming to vary from negative to positive (Figure 4; Figure S9). Nevertheless, if the cost of thermoregulatory behavior increases with mean temperature, behavioral thermoregulation will have little capacity to buffer climate warming (Figure 2b,c, green).

Our model predicts that behavior will have the greatest capacity to ameliorate the detrimental impacts of warming in subtropical latitudes ( $\pm 23.4-40^{\circ}$ latitude, Figure 4; Table S1), paralleling our findings for the subtropical $A$. atra (Figure 3). Here, effective behavioral buffering is predicted in $65 \%$ of subtropical insect species, compared to only $11 \%$ and 
$17 \%$ of species in temperate $\left(> \pm 40^{\circ}\right.$ latitude $)$ and tropical $\left(< \pm 23.4^{\circ}\right.$ latitude) regions, respectively. Of subtropical species, thermoregulatory behavior has the potential to rescue six species (26\% of all subtropical species in the dataset) from local extirpation (Figure 4b). By contrast, behavioral rescue is less likely in temperate and tropical regions where predicted warming produces fewer detrimental impacts (only $22 \%$ and $33 \%$ of temperate and tropical species are forecasted to have reduced performance relative to historical levels, respectively; Figure 4a-d). The effect of behavior was especially low in temperate regions, where greater thermal performance breadths result in weaker benefits of thermoregulation (Figure 4c). Thus, the impact of spatial heterogeneity in the thermal environment is not equally important for all species and geographic regions.

\section{Discussion}

Our framework indicates substantial potential for behavior to mitigate the predicted negative effects of climate warming on animal populations. Yet, predicting which species will benefit the most from thermoregulatory behavior depends on the relationship between the features of the thermal environment, such as the association between thermal variability and mean temperature across ecosystems, and the ability of an organism to utilize the available thermal variability. It seems likely that a positive correlation between the mean environmental temperature and spatial heterogeneity in temperature is common in nature at spatial scales relevant for organisms. Indeed, this relationship has been demonstrated for other taxa and habitats. For example, spatial heterogeneity in an agricultural system increases with mean environmental temperature (Faye, Rebaudo, Carpio, Herrera, \& Dangles, 2017), which reduces the distance required for crop pests to reach a variety of thermal microhabitats (Faye et al., 2017). Similarly, habitats containing thermal refugia - often defined as locations with lower amplitude temperature fluctuations through time (Logan et al., 2013; Scheffers, Edwards, et al., 2014; Scheffers, Evans, et al., 2014) — will become increasingly spatially heterogeneous as $\overline{\mathbb{T}}$ increases because the difference between refugia and the rest of the habitat will correspondingly diverge. Likewise, extreme cold events were accompanied by decreased thermal spatial 
variability for fish in the Everglades National Park, limiting the movement behavior of Common Snook (Boucek, Heithaus, Santos, Stevens, \& Rehage, 2017).

However, it is not merely enough to observe an increase in spatial heterogeneity as environments warm to assume that the costs of behavioral thermoregulation will decrease. This requires understanding of not only how the thermal landscape changes, but also how organisms will utilize the altered landscape, particularly in locations representing environmental extremes (Figure S7). Fully resolving the relationship between $\overline{\mathbb{T}}$ and thermal characteristics, understanding how climate warming may alter such relationships (Caillon et al., 2014), and anticipating how organisms will utilize the new thermal landscape should be a priority for future research.

Our analysis of insect species assumes that benefit functions are representative of the gains in performance made by all individuals of a given insect taxon and that the cost function is a reasonable fit to all individuals regardless of the location or taxon. Most of the thermal performance curves on which our predictions rely are measured in the laboratory by averaging or aggregating across multiple individuals, yet the cost and benefit functions that we derive are based on the energetic gains and losses of individuals. Much has yet to be learned about the importance of intraspecific variation in thermal performance (Moran, Hartig, \& Bell, 2016) and this will surely lead to novel insights on the role of behavior. Furthermore, variation in costs among species, across ecological contexts (Luhring \& DeLong, 2016), and across space and time will undoubtedly affect the potential for behavioral rescue and is an avenue worthy of future research.

While intended to represent a first approximation of the costs of thermoregulatory behavior imposed by the environment, the patterns predicted by our framework are consistent with the movement behavior of organisms in nature. Sears et al. (2016) experimentally manipulated spatial heterogeneity in an open desert habitat and demonstrated that lizards maintain tighter control over their body temperatures and incurred smaller energetic costs in habitats with a greater range, and lower spatial autocorrelation, of environmental temperatures. Furthermore, lizard body temperatures mimicked operative temperatures until reaching a high threshold temperature $\left(\sim 33^{\circ} \mathrm{C}\right)$, at which point thermoregulatory behavior was used to maintain a near constant body temperature even 
as the mean environmental temperature continued to increase. Similarly, Logan et al. (2015) recorded a positive relationship between thermal spatial heterogeneity (standard deviation of temperatures) and activity time of a tropical forest-dwelling lizard species (Anolis lemurinus) and that activity time was much more strongly affected by environmental temperatures above $T_{\text {opt }}$ rather than below it. These results suggest that increased thermal heterogeneity can encourage movement behavior by lowering thermoregulatory costs, even in habitats typically considered to be thermally homogenous.

Although behavior provides a potent defense against rapid environmental change, it has been historically excluded from large-scale climate-impact estimates (Woods et al., 2015). Our results provide a straightforward and general approach for integrating behavior into such estimates. Our framework reveals that the relationship between the thermal features of the environment defines the likelihood for behavioral rescue in a given region. Organisms living in environments where the cost of thermoregulation increases with mean temperature may be particularly vulnerable to the effects of warming. Thus, a conservation priority should be to identify and preserve features of habitats that function to increase thermal heterogeneity as environmental temperatures continue to rise.

Acknowledgments This manuscript was initiated by conversations at the 2016 Gordon Research Conference on Predator-Prey Interactions: New Frontiers in Understanding Predator-Prey Interactions in a Human-Altered World. We thank R.B. Huey for highly constructive criticisms of earlier drafts. This project was completed with permission granted by CapeNature (permit no. AAA007-00206-0056) and approved by the Stellenbosch University Research Ethics Committee (Animal Care and Use protocol number SU-ACUD14-00061).

Conflicts of interest The authors declare there are no competing interests.

Authors' contributions All authors contributed to the development of theory; KA, MLL, and SCT collected empirical data; SBF, DAV, and MIO analyzed data; SBF and DAV wrote the initial draft of the paper and all authors contributed substantially to revisions. 


\section{References}

Adolph, S. C., \& Porter, W. P. (1993). Temperature, activity, and lizard life histories. The American Naturalist, 142(2), 273-295. https://doi.org/10.1086/285538

Angilletta, M. J. (2006). Estimating and comparing thermal performance curves. Journal of Thermal Biology, 31(7), 541-545. https://doi.org/10.1016/j.jther bio.2006.06.002

Angilletta, M. J. (2009). Thermal adaptation: A theoretical and empirical synthesis. Oxford; NY: Oxford University Press.

Basson, C. H., Levy, O., Angilletta, M. J., \& Clusella-Trullas, S. (2017). Lizards paid a greater opportunity cost to thermoregulate in a less heterogeneous environment. Functional Ecology, 31(4), 856-865. https://doi.org/10.1111/1365-2435.12795

Boucek, R. E., Heithaus, M. R., Santos, R., Stevens, P., \& Rehage, J. S. (2017). Can animal habitat use patterns influence their vulnerability to extreme climate events? An estuarine sportfish case study. Global Change Biology, 23(10), 40454057. https://doi.org/10.1111/gcb.13761

Buckley, L. B., Ehrenberger, J. C., \& Angilletta, M. J. (2015). Thermoregulatory behaviour limits local adaptation of thermal niches and confers sensitivity to climate change. Functional Ecology, 29(8), 1038-1047. https://doi. org/10.1111/1365-2435.12406

Caillon, R., Suppo, C., Jérôme Casas, H., Woods, A., \& Pincebourde, S. (2014). Warming decreases thermal heterogeneity of leaf surfaces: Implications for behavioural thermoregulation by arthropods. Functional Ecology, 28(6), 1449-1458. https:// doi.org/10.1111/1365-2435.12288

Dell, A. I., Pawar, S., \& Savage, V. M. (2013). The thermal dependence of biological traits. Ecology, 94(5), 1205-1206. https://doi.org/10.1890/12-2060.1

Deutsch, C. A., Tewksbury, J. J., Huey, R. B., Sheldon, K. S., Ghalambor, C. K., Haak, D. C., \& Martin, P. R. (2008). Impacts of climate warming on terrestrial ectotherms across latitude. Proceedings of the National Academy of Sciences of the United States of America, 105(18), 6668-6672. https://doi.org/10.1073/ pnas.0709472105

Faye, E., Rebaudo, F., Carpio, C., Herrera, M., \& Dangles, O. (2017). Does heterogeneity in crop canopy microclimates matter for pests? Evidence from aerial highresolution thermography. Agriculture, Ecosystems \& Environment, 246(Suppl. C), 124-133.

Fey, S. B., \& Vasseur, D. A. (2016). Thermal variability alters the impact of climate warming on consumer-resource systems. Ecology, 97(7), 1690-1699. https://doi. org/10.1890/15-1838.1

Grant, B. W., \& Dunham, A. E. (1988). Thermally imposed time constraints on the activity of the desert lizard Sceloporus merriami. Ecology, 69(1), 167-176. https://doi.org/10.2307/1943171

Grant, B. W., \& Porter, W. P. (1992). Modeling global macroclimatic constraints on ectotherm energy budgets. American Zoologist, 32(2), 154-178. https://doi. org/10.1093/icb/32.2.154 
Holland, K. N., Brill, R. W., Chang, R. K. C., Sibert, J. R., \& Fournier, D. A. (1992). Physiological and behavioural thermoregulation in bigeye tuna (Thunnus obesus). Nature, 358(6385), 410-412. https://doi.org/10.1038/358410a0

Huang, S.-P., Porter, W. P., Ming-Chung, T. U., \& Chiou, C.-R. (2014). Forest cover reduces thermally suitable habitats and affects responses to a warmer climate predicted in a high-elevation lizard. Oecologia, 175(1), 25-35. https://doi. org/10.1007/s00442-014-2882-1

Huey, R. B. (1974). Behavioral thermoregulation in lizards: Importance of associated costs. Science (New York, N.Y.), 184(4140), 1001-1003. https://doi.org/10.1126/ science.184.4140.1001

Huey, R. B., Hertz, P. E., \& Sinervo, B. (2003). Behavioral drive versus behavioral inertia in evolution: A null model approach. The American Naturalist, 161(3), 357-366. https://doi.org/10.1086/346135

Huey, R. B., Kearney, M. R., Krockenberger, A., Holtum, J. A. M., Jess, M., \& Williams, S. E. (2012). Predicting organismal vulnerability to climate warming: Roles of behaviour, physiology and adaptation. Philosophical Transactions of the Royal Society B: Biological Sciences, 367(1596), 1665-1679. https://doi.org/10.1098/ $\underline{\text { rstb. } 2012.0005}$

Huey, R. B., \& Slatkin, M. (1976). Cost and benefits of lizard thermoregulation. The Quarterly Review of Biology, 51(3), 363-384. https://doi.org/10.1086/409470

Kearney, M., \& Porter, W. (2009). Mechanistic niche modelling: Combining physiological and spatial data to predict species' ranges. Ecology Letters, 12(4), 334-350. https://doi.org/10.1111/j.1461-0248.2008.01277.x

Kearney, M., Shine, R., \& Porter, W. P. (2009). The potential for behavioral thermoregulation to buffer 'cold-blooded' animals against climate warming. Proceedings of the National Academy of Sciences of the United States of America, 106(10), 3835-3840. https://doi.org/10.1073/pnas.0808913106

Litchman, E., \& Klausmeier, C. A. (2008). Trait-based community ecology of phytoplankton. Annual Review of Ecology, Evolution, and Systematics, 39(1), 615639. https://doi.org/10.1146/annur ev.ecolsys.39.110707.173549

Logan, M. L., Fernandez, S. G., \& Calsbeek, R. (2015). Abiotic constraints on the activity of tropical lizards. Functional Ecology, 29(5), 694-700. https://doi. org/10.1111/1365-2435.12379

Logan, M. L., Huynh, R. K., Precious, R. A., \& Calsbeek, R. G. (2013). The impact of climate change measured at relevant spatial scales: New hope for tropical lizards. Global Change Biology, 19(10), 3093-3102. https://doi.org/10.1111/gcb.12253

Long, R. A., Terry Bowyer, R., Porter, W. P., Mathewson, P., Monteith, K. L., \& Kie, J. G. (2014). Behavior and nutritional condition buffer a large-bodied endotherm against direct and indirect effects of climate. Ecological Monographs, 84(3), 513532. https://doi.org/10.1890/13-1273.1

Luhring, T. M., \& DeLong, J. P. (2016). Predation changes the shape of thermal performance curves for population growth rate. Current Zoology, 62(5), 501-505. https://doi.org/10.1093/cz/zow045 
Martin, T. L., \& Huey, R. B. (2008). Why 'suboptimal' is optimal: Jensen's inequality and ectotherm thermal preferences. The American Naturalist, 171(3), E102-118. https://doi.org/10.1086/527502

Moran, E. V., Hartig, F., \& Bell, D. M. (2016). Intraspecific trait variation across scales: Implications for understanding global change responses. Global Change Biology, 22(1), 137-150. https://doi.org/10.1111/gcb.13000

Pincebourde, S., Murdock, C. C., Vickers, M., \& Sears, M. W. (2016). Fine-scale microclimatic variation can shape the responses of organisms to global change in both natural and urban environments. Integrative and Comparative Biology, 56(1), 45-61. https://doi.org/10.1093/icb/icw016

Porter, W. P., \& Gates, D. M. (1969). Thermodynamic equilibria of animals with environment. Ecological Monographs, 39(3), 227-244. https://doi. org/10.2307/1948545

Porter, W. P., \& Kearney, M. (2009). Size, shape, and the thermal niche of endotherms. Proceedings of the National Academy of Sciences of the United States of America, 106(Suppl. 2), 19666-19672. https://doi.org/10.1073/pnas.0907321106

Porter, W. P., Mitchell, J. W., Beckman, W. A., \& DeWitt, C. B. (1973). Behavioral implications of mechanistic ecology. Oecologia, 13(1), 1-54. https://doi. org/10.1007/BF00379617

Rusch, T. W., \& Angilletta, M. J. (2017). Competition during thermoregulation altered the body temperatures and hormone levels of lizards. Functional Ecology, 31(8), 1519-1528. https://doi.org/10.1111/1365-2435.12869

Scheffers, B. R., Edwards, D. P., Diesmos, A., Williams, S. E., \& Evans, T. A. (2014). Microhabitats reduce animal's exposure to climate extremes. Global Change Biology, 20(2), 495-503. https://doi.org/10.1111/gcb.12439

Scheffers, B. R., Evans, T. A., Williams, S. E., \& Edwards, D. P. (2014). Microhabitats in the tropics buffer temperature in a globally coherent manner. Biology Letters, 10(12), 20140819. https://doi.org/10.1098/rsbl.2014.0819

Sears, M. W., \& Angilletta, M. J. (2015). Costs and benefits of thermoregulation revisited: Both the heterogeneity and spatial structure of temperature drive energetic costs. The American Naturalist, 185(4), E94-E102. https://doi. org $/ 10.1086 / 680008$

Sears, M. W., Angilletta, M. J., Schuler, M. S., Borchert, J., Dilliplane, K. F., Stegman, M., ... Mitchell, W. A. (2016). Configuration of the thermal landscape determines thermoregulatory performance of ectotherms. Proceedings of the National Academy of Sciences of the United States of America, 113(38), 10595-10600. https://doi.org/10.1073/pnas.1604824113

Sears, M. W., Raskin, E., \& Angilletta, M. J. (2011). The world is not flat: Defining relevant thermal landscapes in the context of climate change. Integrative and Comparative Biology, 51(5), 666-675. https://doi.org/10.1093/icb/icr111

Shine, R., \& Kearney, M. (2001). Field studies of reptile thermoregulation: How well do physical models predict operative temperatures? Functional Ecology, 15(2), 282-288. https://doi.org/10.1046/j.1365-2435.2001.00510.x 
Sinervo, B., Méndez-de-la-Cruz, F., Miles, D. B., Heulin, B., Bastiaans, E., Cruz, M.-S., ... Sites, J. W. (2010). Erosion of lizard diversity by climate change and altered thermal niches. Science, 328(5980), 894-899. https://doi.org/10.1126/ science.1184695

Sunday, J. M., Bates, A. E., Kearney, M. R., Colwell, R. K., Dulvy, N. K., Longino, J. T., \& Huey, R. B. (2016). Thermal-safety margins and the necessity of thermoregulatory behavior across latitude and elevation. Proceedings of the National Academy of Sciences of the United States of America, 111(15), 5610-5615. https://doi. org/10.1073/pnas.1316145111

Thomas, M. K., Aranguren-Gassis, M., Kremer, C. T., Gould, M. R., Anderson, K., Klausmeier, C. A., \& Litchman, E. (2017). Temperature- nutrient interactions exacerbate sensitivity to warming in phytoplankton. Global Change Biology, 23(8), 3269-3280. https://doi.org/10.1111/gcb.13641

Thomas, M. K., Kremer, C. T., Klausmeier, C. A., \& Litchman, E. (2012). A global pattern of thermal adaptation in marine phytoplankton. Science, 338(6110), 1085-1088. https://doi.org/10.1126/science.1224836

Vasseur, D. A., DeLong, J. P., Gilbert, B., Greig, H. S., Harley, C. D. G., McCann, K. S., ... O'Connor, M. I. (2014). Increased temperature variation poses a greater risk to species than climate warming. Proceedings of the Royal Society of London B: Biological Sciences, 281(1779), 20132612. https://doi.org/10.1098/ rspb.2013.2612

Waldschmidt, S. R., Jones, S. M., \& Porter, W. P. (1986). The effect of body temperature and feeding regime on activity, passage time, and digestive coefficient in the lizard Uta stansburiana. Physiological Zoology, 59(3), 376-383. https://doi. org/10.1086/physz ool.59.3.30156109

Williams, S. E., Shoo, L. P., Isaac, J. L., Hoffmann, A. A., \& Langham, G. (2008). Towards an integrated framework for assessing the vulnerability of species to climate change. PLOS Biology, 6(12), e325. https://doi.org/10.1371/journal. pbio. 0060325

Woods, H. A., Dillon, M. E., \& Pincebourde, S. (2015). The roles of microclimatic diversity and of behavior in mediating the responses of ectotherms to climate change. Journal of Thermal Biology, 54, 86-97. https://doi.org/10.1016/j. jtherbio.2014.10.002

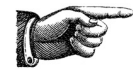

\section{Supporting Materials follow.}




\section{Supporting Materials}

\section{Opportunities for Behavioral Rescue Under Rapid Environmental Change}

\section{Index:}

1. Additional information on defining the cost structure (Appendix 1; Fig. S1-S7).

2. Additional detail on the interactions between mean costs and cost scaling (Fig. S8).

3. Summaries of performance estimates of species by geographic region (Table S1).

4. Species specific responses of insect performance in response to climate change (Fig. S9).

5. Estimated mean performance of Agama atra lizards (Fig. S10) and associated TPC estimates (Table S2)

6. Comparisons of Agama atra territories (Fig. S11) and accompanying photographs (Fig. S12).

7. Spatial heterogeneity of Agama atra territories (Fig. S13) and estimated cost parameters associated with each territory (Fig. S15).

8. The relationship between spatial standard deviation in Agama atra territories, depending on the range of temperatures that A. atra can tolerate (Fig. S14).

9. Supplemental methods for characterizing Agama atra territories and thermal sensitivity (Appendix 2). 


\section{SI Appendix 1: Defining the cost structure of thermoregulatory behavior}

In this appendix we explore how the shape of the thermoregulatory behavior cost function changes in response to properties of the thermal environment, $\mathbb{T}$, and the individual that is behaviorally thermoregulating. The purpose of this appendix is to establish a reasonable approximation for the shape of the cost function and to understand how specific aspects of $\mathbb{T}$ and the behaviorally thermoregulating organism impact the overall shape and steepness of the cost function.

In the examples below, we elaborate on the specific details involved with each scenario. In each case, the cost of achieving a body temperature is determined by the energetic costs associated with maintaining a particular body temperature, given $\mathbb{T}$. Similar to previous studies [1,2], we define the cost of maintaining a particular body temperature as being linearly proportional to the distance needed to travel to achieve this temperature. This accounts for the energetic costs associated with moving, potential threats faced from predation, and lost foraging opportunities incurred while traveling.

Unless otherwise specified, the $\mathbb{T}$ is defined as a thermal environment with mean $\mu=$ $25^{\circ} \mathrm{C}$, standard deviation $\sigma=3$, and moderate spatial autocorrelation $(\gamma=-3)$. The thermal landscape is defined by a 256 × 256 two-dimensional grid folded as a torus. Unless otherwise specified, the costs associated with achieving a particular body temperature are defined by the mean distance an organism must travel to maintain a particular body temperature. This value is calculated from simulating movement dynamics with 1000 simulations of a particular $\mathbb{T}$ and a random starting location. This process simulates the following ecological conditions: 1) the spatial configuration of temperatures will change through time and organisms have to constantly seek out a preferred temperature as this change occurs, 2) organisms will have to leave locations of preferred temperatures for resource access before navigating back to their preferred locations.

Unless otherwise specified, the individual uses a simple model of adaptive movement where it senses the local environment (current environment plus the eight surrounding locations), assesses the vertical and horizontal gradient in temperatures independently, and moves in the correct direction (e.g., the direction that reduces the difference between their current and desired body temperature) with $90 \%$ certainty. After a threshold number of time steps have elapsed $(t=6,500$, which represents $\sim 10 \%$ of the pixels on the landscape), the organism will stop searching for a particular temperature if it has not already found a cell that is within $+/-1$ degree of its preferred temperature.

Below we use the aforementioned approach to understand the importance of specific properties of the environment and organism for the costs of behavioral thermoregulation. 


\section{1) The effect of spatial autocorrelation}

To investigate the role of spatial autocorrelation for the cost of thermoregulation, we simulated three classes of $\mathbb{T}$ : spatially uncorrelated ( $\gamma=0$, Fig. S1 top left), moderate spatial autocorrelation ( $\gamma=-3$, top center), and high spatial autocorrelation $(\gamma=-6$, top right). The landscapes are otherwise identical in their mean and standard deviation in temperature. In all instances, a positive second derivative function with a minimum temperature of $25^{\circ} \mathrm{C}$ defines the cost structure measured by our simulated movement of individuals (Figure S1 bottom row, black lines); however, the steepness of the cost function away from this minimum and the value of the cost function minima increased with spatial autocorrelation. As such, at the scale of the entire landscape, uncorrelated environments lower the cost of thermoregulatory behavior.

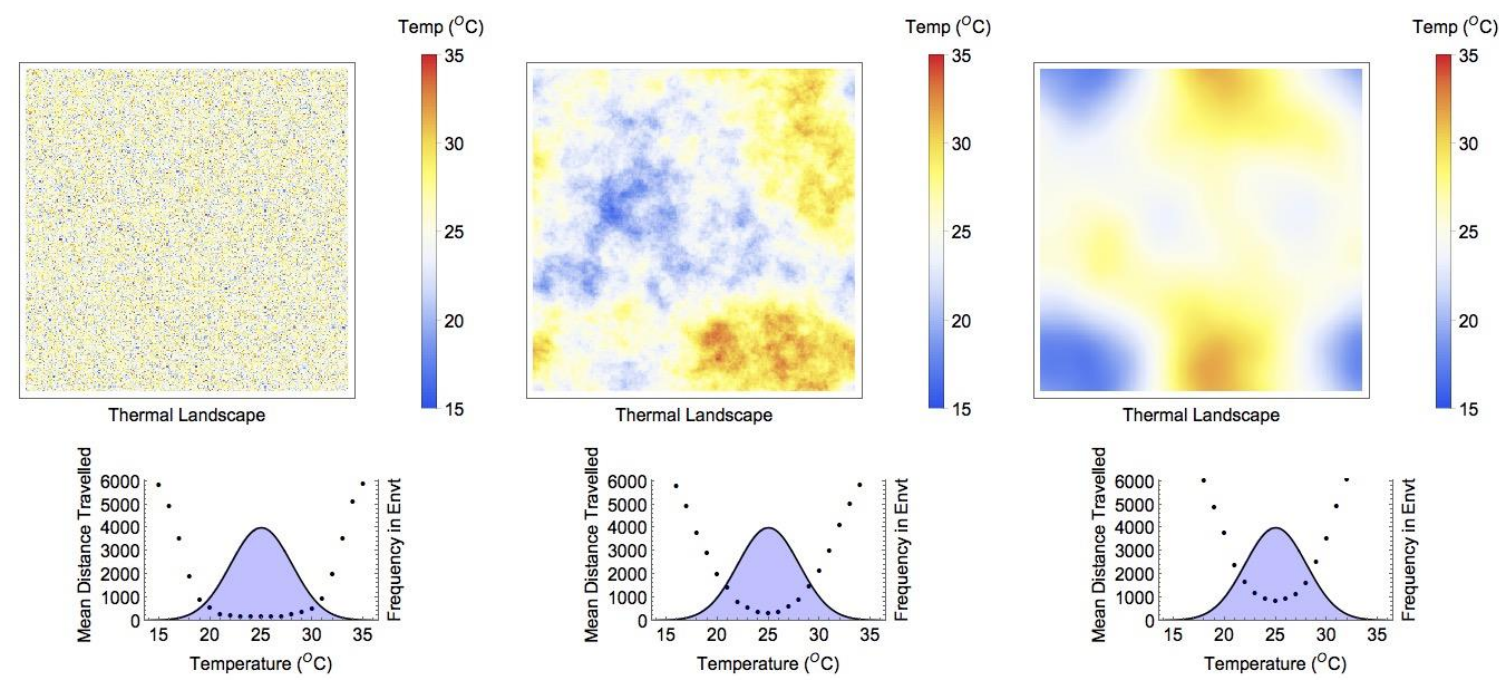

Figure S1: Effects of spatial autocorrelation on the costs of behavioral thermoregulation. Top row: examples of spatially uncorrelated $(\gamma=0$, left), moderate spatial autocorrelation $(\gamma=-3$, center), and high spatial autocorrelation landscapes $(\gamma=-6$, right). Bottom row: the costs of achieving body temperature (black dots) calculated as the mean of 1000 simulations measuring the distance traveled from random starting locations to achieve each temperature. The blue shading is a frequency distribution of the available environmental temperatures from which each landscape is formed. 


\section{2) The effect of thermal heterogeneity}

To investigate the role of thermal heterogeneity in environmental temperature for the cost of thermoregulation, we simulated three classes of thermal landscapes: low variation in environmental temperature ( $\sigma=1.5$, Fig. S2 top left), moderate variation in environmental temperature ( $\sigma=3$, top center), and high variation in environmental temperature ( $\sigma=4.5$, top right). In all instances, the positive second derivative function with a minimum temperature of $25^{\circ} \mathrm{C}$ defined the cost structure. However, the steepness of the cost function decreased as thermal heterogeneity increased (Figure S2). The cost of thermoregulatory behavior is lower in thermally heterogeneous environments

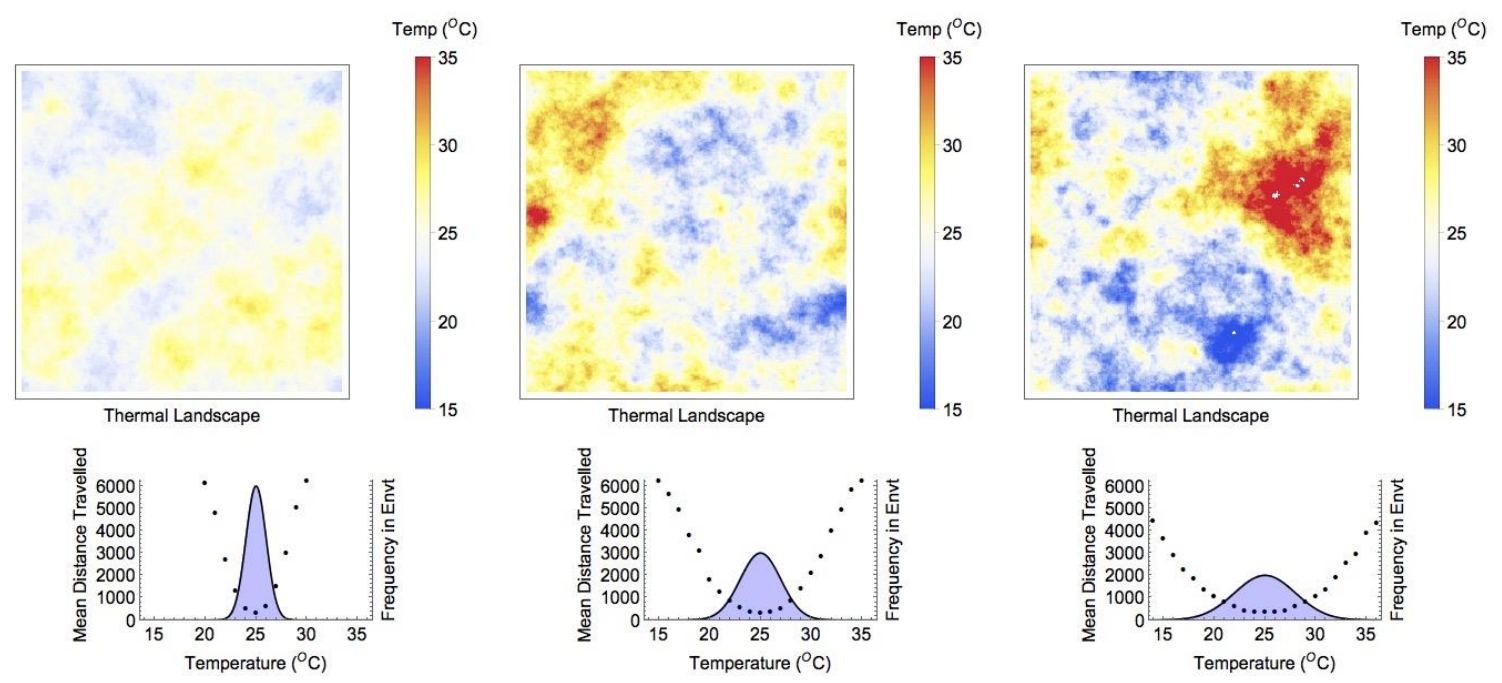

Figure S2: Effects of thermal heterogeneity on the cost of behavioral thermoregulation. Top row: examples of low $(\sigma=1.5$, left), moderate $(\sigma=3$, center), and high $(\gamma=4.5$, right) heterogeneity in the thermal landscape. Bottom row: the costs of achieving body temperature (black dots) calculated as the mean of 1000 simulations measuring the distance traveled from a random starting location to each temperature. The blue shading bounded by black solid line is a frequency distribution of the available environmental temperatures from which each individual landscape is formed. 


\section{3) The effects of behavioral thermoregulation movement strategy}

To investigate the role of thermoregulatory strategy in the cost of thermoregulation, we simulated three movement strategies in identical thermal landscapes: omniscient searching (Fig. S3 left) defined as an individual traveling the shortest Manhattan distance from the starting location to its desired temperature, locally-informed searching (center), defined by the individual making a probabilistic move towards their desired temperature based on the immediately adjacent environmental temperatures, and random searching (right) defined by the organism moving from a starting location in a random direction. In all instances, the positive second derivative function with the minima $25^{\circ} \mathrm{C}$ defined the cost structure; however, steepness of the cost function greatly increases as the organism exhibits reduced searching efficiency (Figure S3 bottom row, black lines). Thus, the costs of thermoregulatory behavior will be reduced for more efficient searchers.

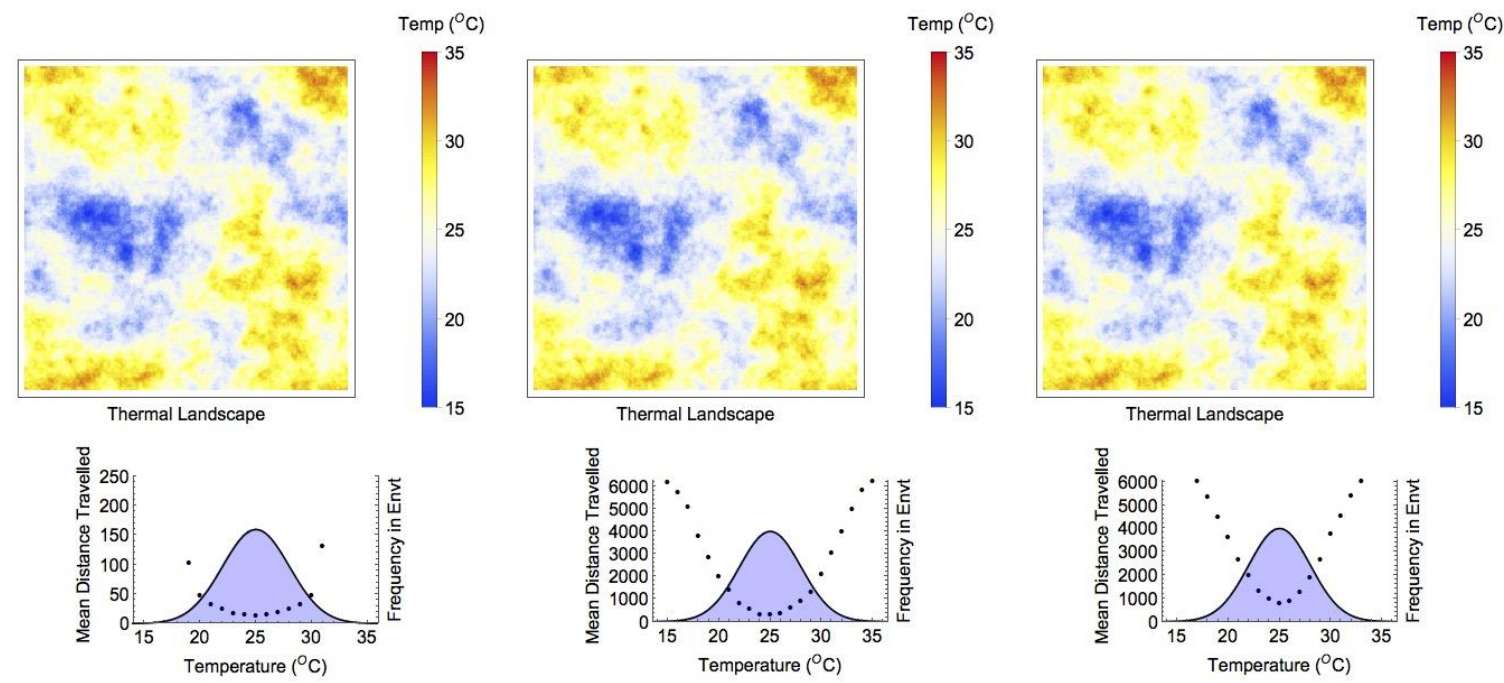

Figure S3: Effects of movement strategy on the costs of behavioral thermoregulation. Top row: the thermal landscapes that omniscient (left), locally-informed (center), and random (right) searching individuals inhabit are identical. Bottom row: the costs of achieving body temperature (black dots) calculated as the mean of 1000 simulations measuring the distance traveled from random starting locations to each temperature, based on the aforementioned search algorithms. Note different scaling of y-axis for the bottom left panel. The blue shading is a frequency distribution of the available environmental temperatures from which each individual landscape is formed, which are identical among landscapes. 


\section{4) The effect of individual starting location}

In the above scenarios, the mean search distance traveled is calculated as the mean distance traveled from a set of random starting locations to a desired temperature. However, it is possible that an individual will inhabit an environment where it is consistently moving from a particular temperature (e.g., an underground burrow, or a thermal environment rich in a key resource) to achieve a particular body temperature. In such instances, we can define the cost of thermoregulatory behavior as the distance traveled from starting locations having particular temperatures (e.g., cooler microsites) to locations with a different, preferred temperature (e.g., warmer microsites). In order to investigate the role of starting location for the cost of thermoregulatory behavior, we simulated three movement strategies in identical thermal landscapes: starting from a comparatively cool location of $22^{\circ} \mathrm{C}$ (Fig. S4, left), starting from a random location (center), and starting from a comparatively warm location $\left(28^{\circ} \mathrm{C}\right.$, right). When the starting location is not the most abundant temperature in the environment (Fig. S4 right and left), the cost function becomes asymmetric and is skewed in the direction of where a greater abundance of such thermal habitats exist.

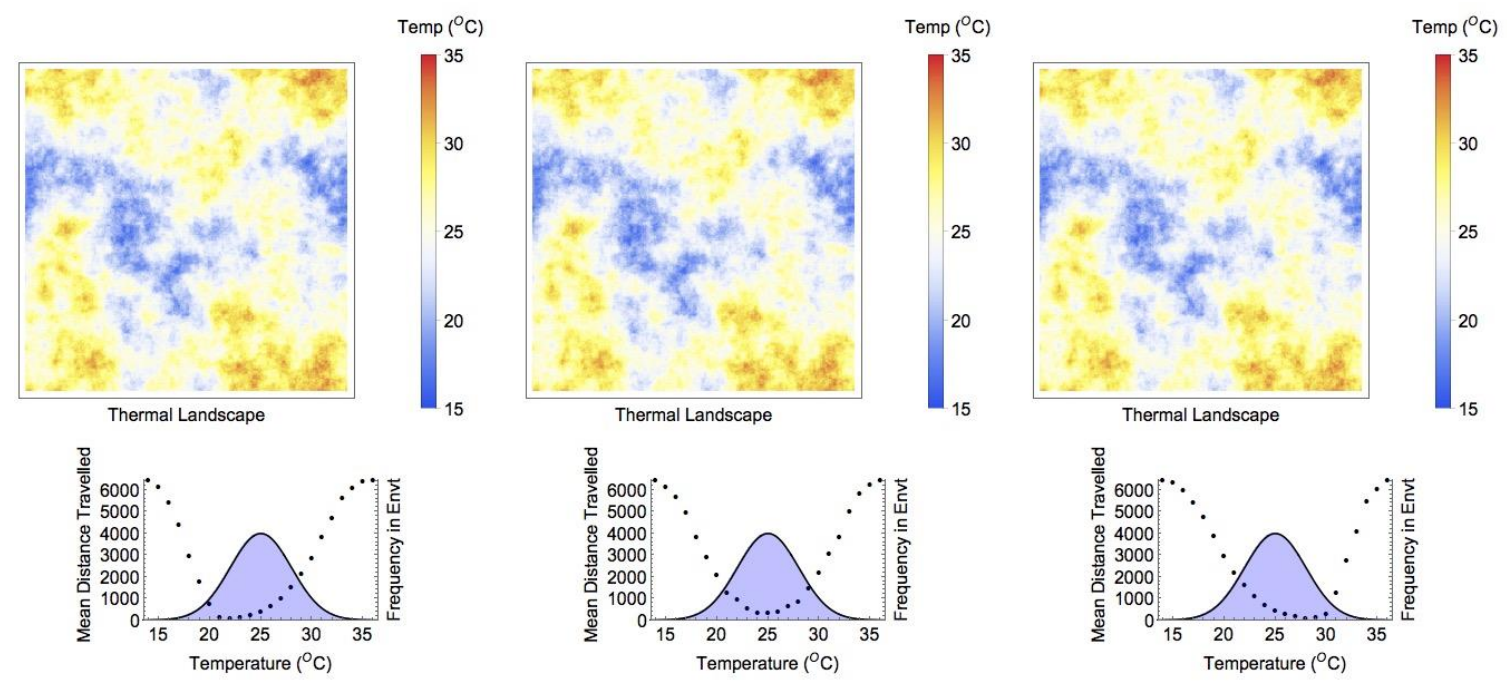

Figure S4: Effects of starting location on the costs of thermoregulatory behavior. Top row: individuals starting from comparatively cool temperature (left), random (center), or comparatively warm (right) starting locations thermoregulate in identical habitats. Bottom row: the costs of achieving body temperature (black dots) calculated as the mean of 1000 simulations measuring the distance traveled. The blue shading is a frequency distribution of the available environmental temperatures from which each individual landscape is formed, which is identical among landscapes. 


\section{5) The effect of bimodal thermal environments}

In the aforementioned scenarios, we consider the cost of thermoregulation in environments with unimodal temperature distributions. Here, we consider the costs of thermoregulation in bimodal thermal environments (Figure S5, top center and top right panels). Both environments are mixtures of $\mu=30^{\circ} \mathrm{C}, \sigma=2$ and $\mu=20^{\circ} \mathrm{C}, \sigma=2$ distributions; Figure S5 center panel draws 75\% from the distribution with the high mean whereas the Figure S5 right panel draws 75\% from the distribution with the lower mean (Figure S5, bottom row blue shading). While the cost function of the unimodal thermal environment (Figure S5 left) exhibits a single minimum value occurring at the mean of the distribution, the cost functions for the bimodal environments exhibit two minima, each located at the two means from the bimodal distribution. While the mean environmental temperature coincides with the minimum of the cost function in a normally distributed environment, the mean environmental temperature in bi- and multimodal thermal environments can coincide with regions on the cost function that are not the minima of the cost function. As such, non-normally distributed thermal environments strongly favor a divergence between mean ambient temperature and body temperature, as the cost of thermoregulatory behavior is reduced when body temperatures deviate from the mean in these environments.

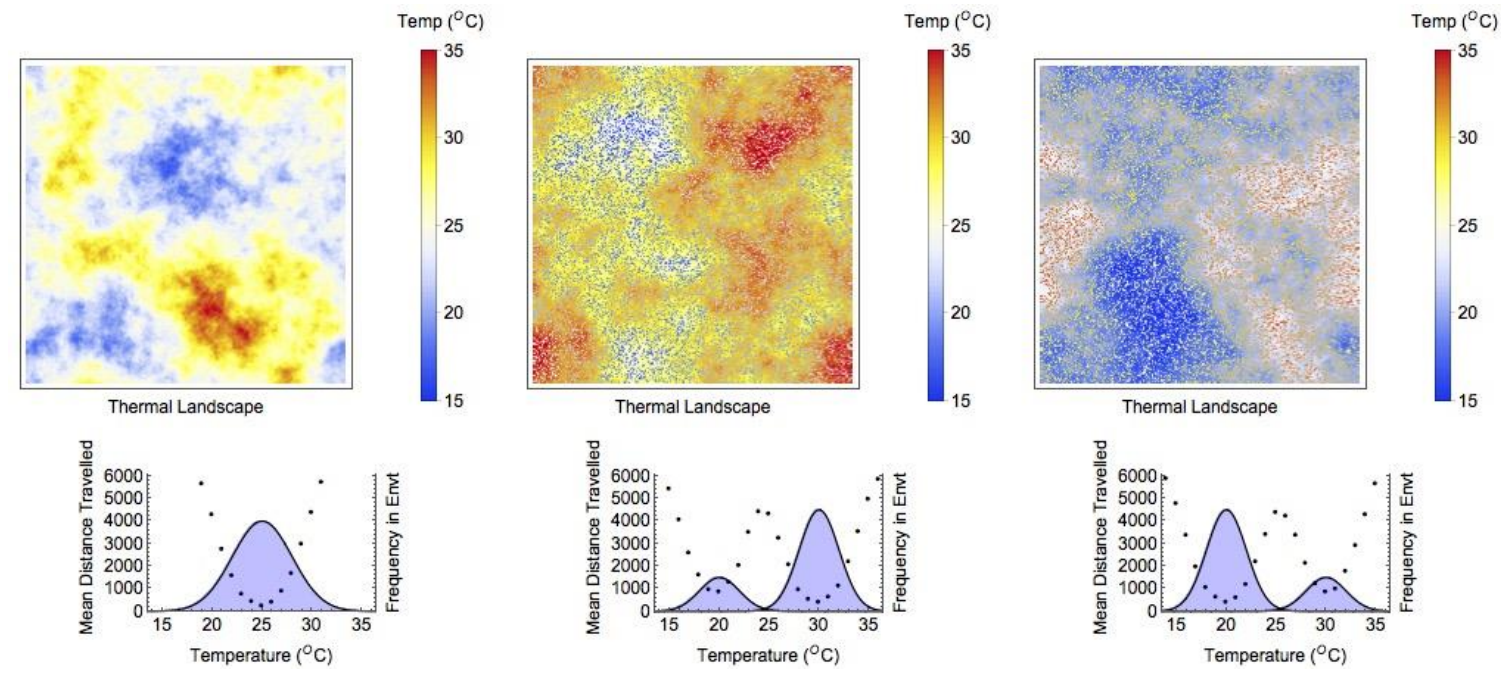

Figure S5: Effects of bimodal temperature distributions on the costs of thermoregulation. Top row: a normally distributed thermal environment (left), a bimodal thermal environment emphasizing high temperatures (middle), and a bimodal thermal environment emphasizing low temperatures (right). Bottom row: the costs of achieving body temperature (black dots) calculated as the mean of 1000 simulations measuring the distance traveled from random starting locations to each temperature. The blue shading is a frequency distribution of the available environmental temperatures from which each individual landscape is formed. 


\section{6) The effect of uniform versus normal temperature distributions}

Above, we examined the cost of thermoregulation in environments drawn from normally distributed temperature distributions. Here, we consider the costs of thermoregulation in an environment with a uniform distribution of temperatures (Figure S6, top left). The cost function of thermoregulation in the uniformly distributed thermal environment (Figure S6, lower left) exhibits a single minimum value occurring at the mean of the distribution, similar to the cost function associated with the normally distributed environment. However, the steepness of the cost function is reduced when temperatures are uniformly distributed. As such, the rarity of particular thermal environments (e.g., the low and high temperature environments when temperatures are normally distributed) is not a necessary condition for producing the concave shape of the cost function.
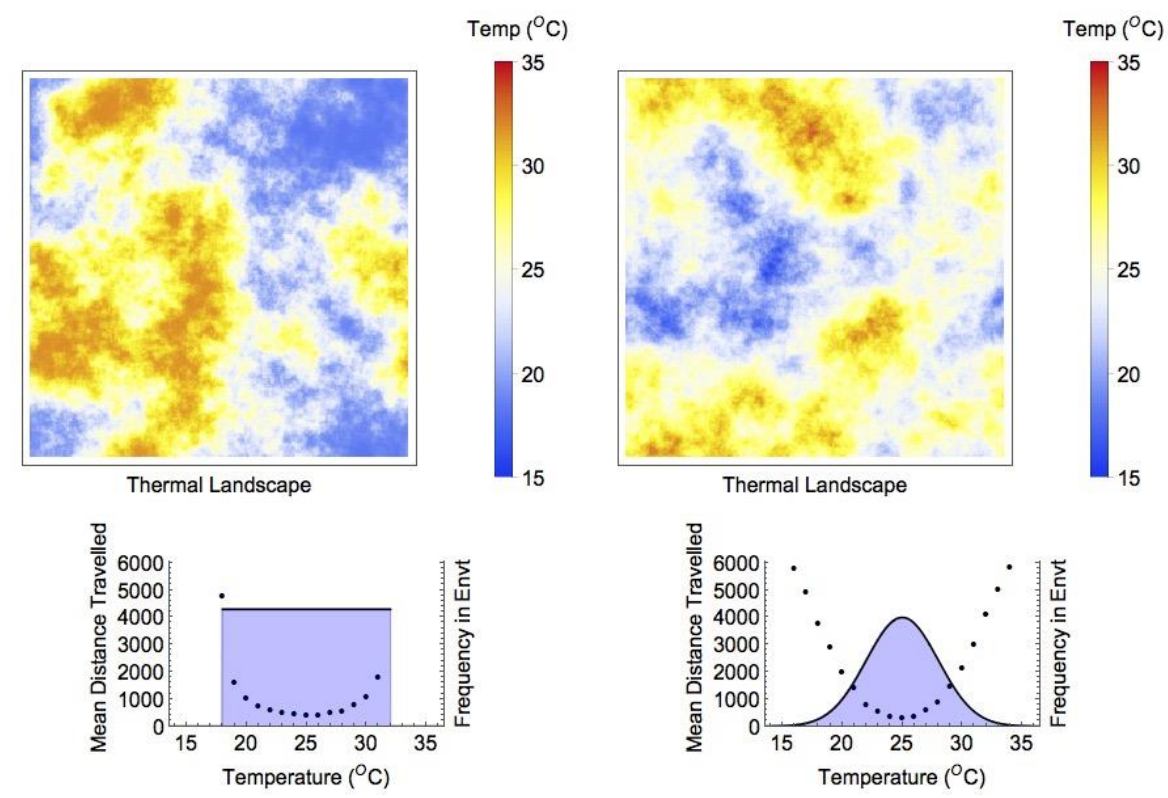

Figure S6: Effects of uniform versus normal temperature distribution on the cost of thermoregulatory behavior. Top row: examples of a uniform (left) and a normal temperature distribution (right) with similar levels of autocorrelation. Bottom row: the costs of achieving body temperature (black dots) calculated as the mean of 1000 simulations measuring the distance traveled from random starting locations to each temperature for uniform (bottom left) and normally distributed environments (bottom right). The blue shading is a frequency distribution of the available environmental temperatures from which each individual landscape is formed. 


\section{7) The effect of not being able to move across all existing environmental conditions}

Previously (Fig. S1-S6), we assume that the individual is capable of moving across all regions of the thermal habitat in order to arrive at a specific area, including the ability to cross particularly hot regions of the landscape. However, specific locations of the environment may be functionally unavailable to an organism if these areas exceed a particular temperature. As such, below we consider the costs of thermoregulation in three identical environments (Figure S7 left, center, right), where the individual cannot to move across locations above $30^{\circ} \mathrm{C}$ (left), locations at or above $25^{\circ} \mathrm{C}$ (center), or locations above $23^{\circ} \mathrm{C}$ (right). In these instances, a new pattern emerges depending on how extreme the environmental conditions are for the individual. If we assume in this example that an organism cannot access environments (even temporarily) above its $\mathrm{CT}_{\max }$, the left panel represents conditions where the modal environmental temperature is lower than $\mathrm{CT}_{\max }$. In this instance, irrespective of searching for temperatures above or below the modal temperature, the cost function (red dots) is almost identical to the cost function where an individual can access all temperatures (black dots). However, in the instance where the modal temperature approximates (center panel) or exceeds (right panel) the modal temperature, the mean cost of locating temperatures increases and the cost function becomes asymmetric relative to scenarios where the individual can move across all temperatures.

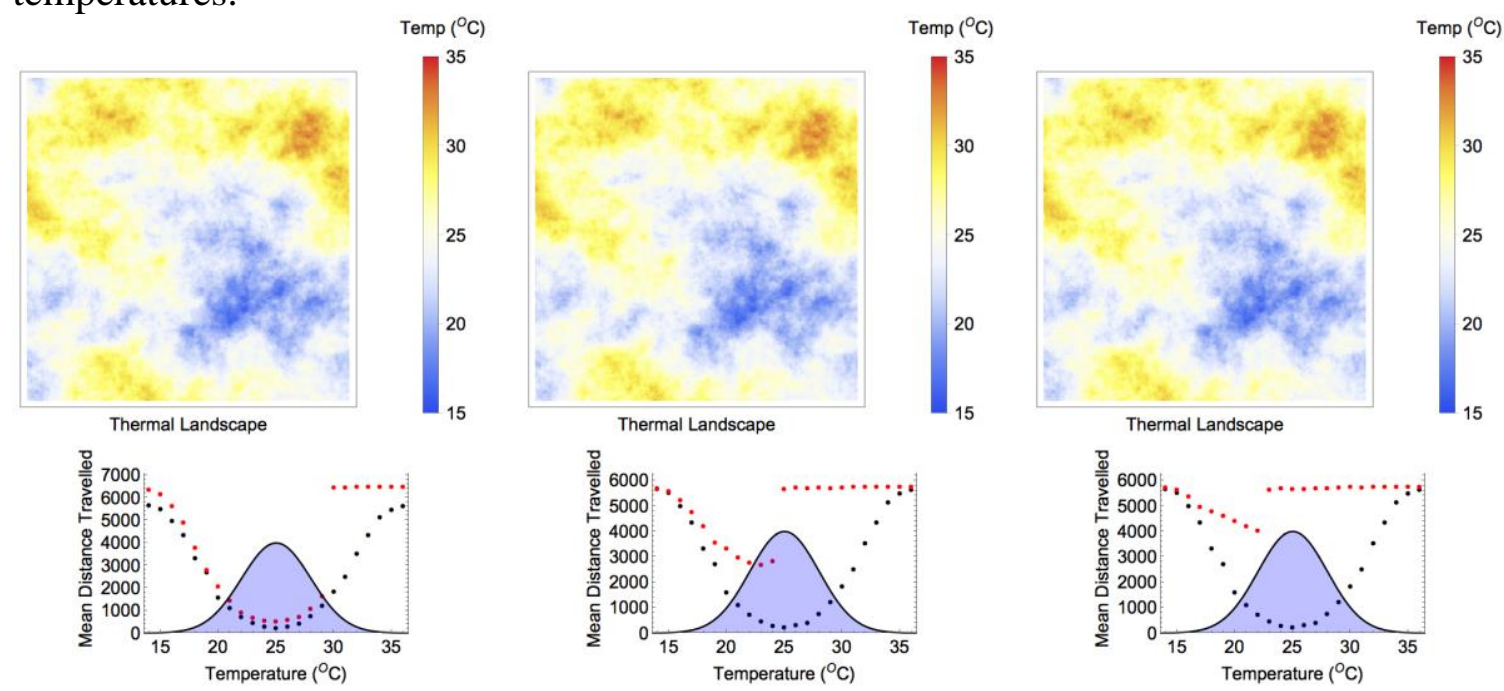

Figure S7: Effects of an individual being restricted from moving into hot regions of the landscape on the cost of thermoregulatory behavior. Top row: three identical landscapes that are inhabited by a hypothetical organism that cannot move into regions (for any amount of time) that exceed $30^{\circ} \mathrm{C}$ (left), $25^{\circ} \mathrm{C}$ (center), or $23^{\circ} \mathrm{C}$ (left). Bottom row: the movement costs of achieving body temperatures (red dots) calculated as the mean of 1000 simulations measuring the distance traveled from random starting locations to each temperature, based on being restricted from moving into regions exceeding particular temperatures (defined in the top row). Black dots represent the search distances for an organism that can access all environmental temperatures. The blue shading is a frequency distribution of the available environmental temperatures from which each individual landscape is formed. 


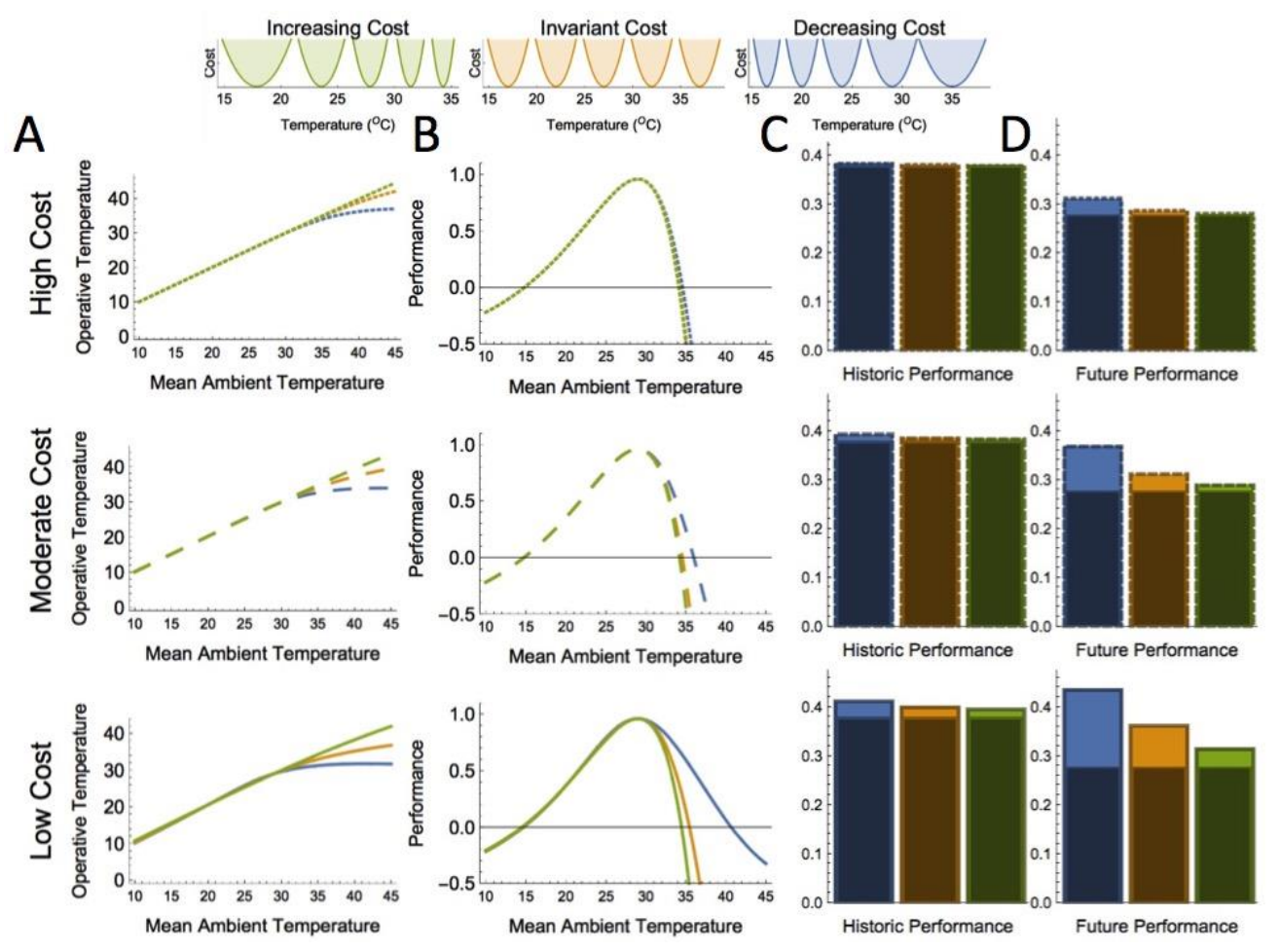

Figure S8: The extent to which behavior modifies population fitness depends on the cost of behavioral thermoregulation. A) Predicted body temperatures and B) and corresponding performance for a hypothetical organism based on environments with different mean costs of thermoregulation (dotted lines are high cost environments, top row; dashed lines are medium cost environments, middle row; solid lines are low cost environments, bottom row). Line color indicates whether the cost of thermoregulation increases (green), is invariant (yellow), or decreases (blue) with increasing mean temperature (see top panel). C) Mean performance of 38 insect species given the cost based on historic and (D) 2050 climate scenarios (see methods), where dark shading represents the performance estimate without behavior and light shading indicates the extent to which behavior can increase performance. 
Table S1: The percentage of temperate, subtropical, and tropical species from Fig. 3 (the instance of low average costs and costs decreasing as $\overline{\mathbb{T}}$ increases) exhibiting particular behavioral features. $W_{f}=$ future performance with no behavior, $W_{h}=$ historic performance with no behavior, $W_{f b}=$ future performance with behavior, $W_{h b}=$ historic performance with behavior.

\begin{tabular}{|l|l|l|l|l|}
\hline Process & Definition & $\begin{array}{l}\% \text { Temperate } \\
(\mathrm{n}=9)\end{array}$ & $\begin{array}{l}\% \text { Subtropical } \\
(\mathrm{n}=23)\end{array}$ & $\begin{array}{l}\text { \% Tropical } \\
(\mathrm{n}=6)\end{array}$ \\
\hline $\begin{array}{l}\text { Behavior enhances } \\
\text { performance in future climates }\end{array}$ & $W_{f b}>W_{f}$ & $22 \%$ & $100 \%$ & $67 \%$ \\
\hline $\begin{array}{l}\text { Negative impact of warming } \\
\text { without behavior }\end{array}$ & $W_{h}>W_{f}$ & $22 \%$ & $65 \%$ & $33 \%$ \\
\hline $\begin{array}{l}\text { Behavioral buffering is } \\
\text { predicted to occur }\end{array}$ & $\begin{array}{l}\text { When } W_{h}>W_{f}, \\
W_{f b}>W_{f}\end{array}$ & $11 \%$ & $65 \%$ & $17 \%$ \\
\hline $\begin{array}{l}\text { Behavioral rescue is predicted } \\
\text { to occur }\end{array}$ & $\begin{array}{l}\text { When } W_{h}>W_{f}, \\
W_{f b}>0>W_{f}\end{array}$ & $0 \%$ & $26 \%$ & $0 \%$ \\
\hline
\end{tabular}



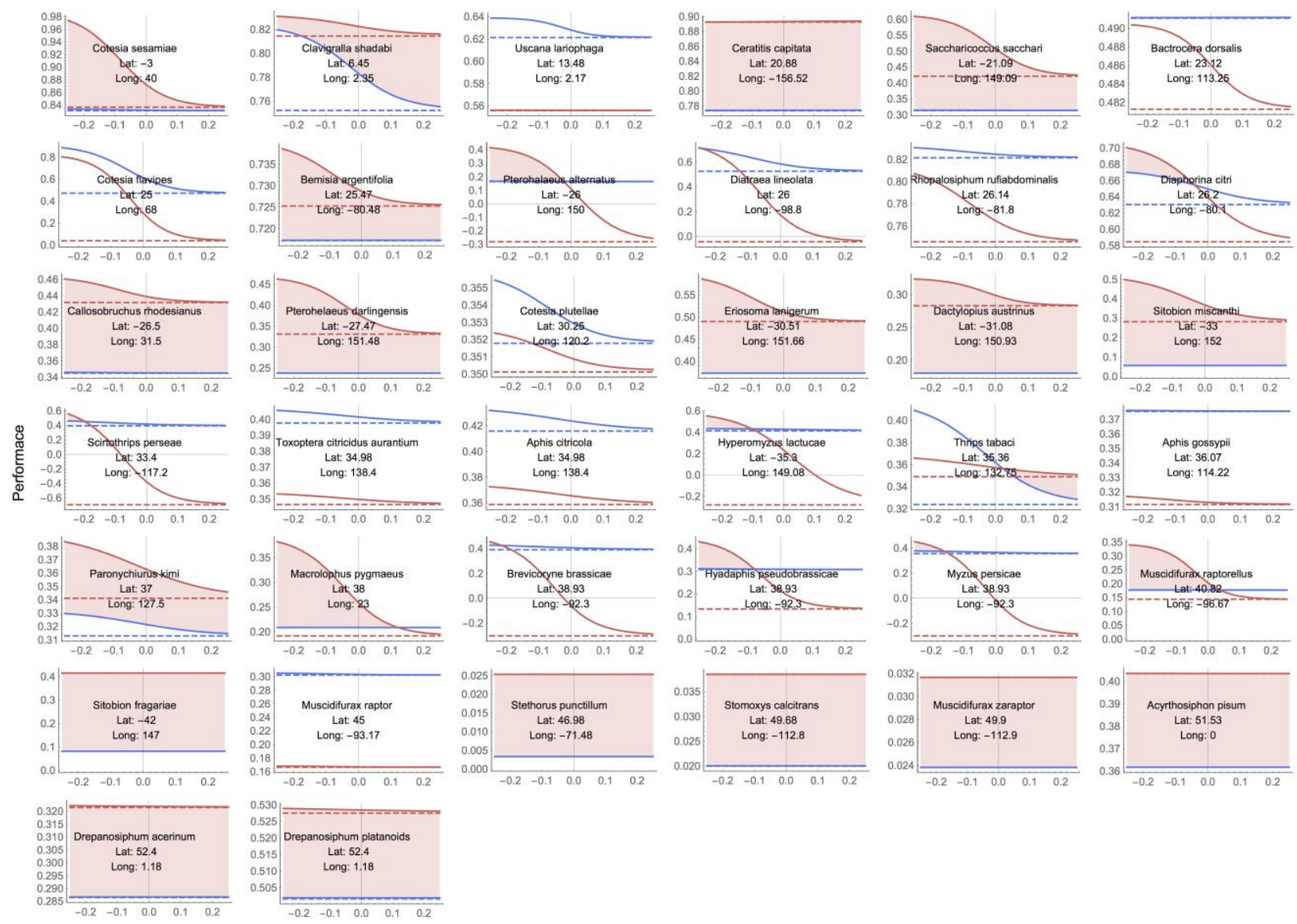

Figure S9: Detailed predictions of insect performance in response to climate warming. Each panel represents a species of insect of which three examples are presented in the main text (Fig. 4). Dashed lines represent performance estimates without behavior in historic (dashed blue) and 2050 (dashed red) environments; solid lines represent performance estimates allowing behavior in historic (solid blue) and 2050 (solid red) environments. Negative $\mathrm{x}$-axis values indicate a negative relationship between mean temperature and the cost of thermoregulation (e.g., the costs of behavioral thermoregulation decrease as mean environmental temperature increases). 


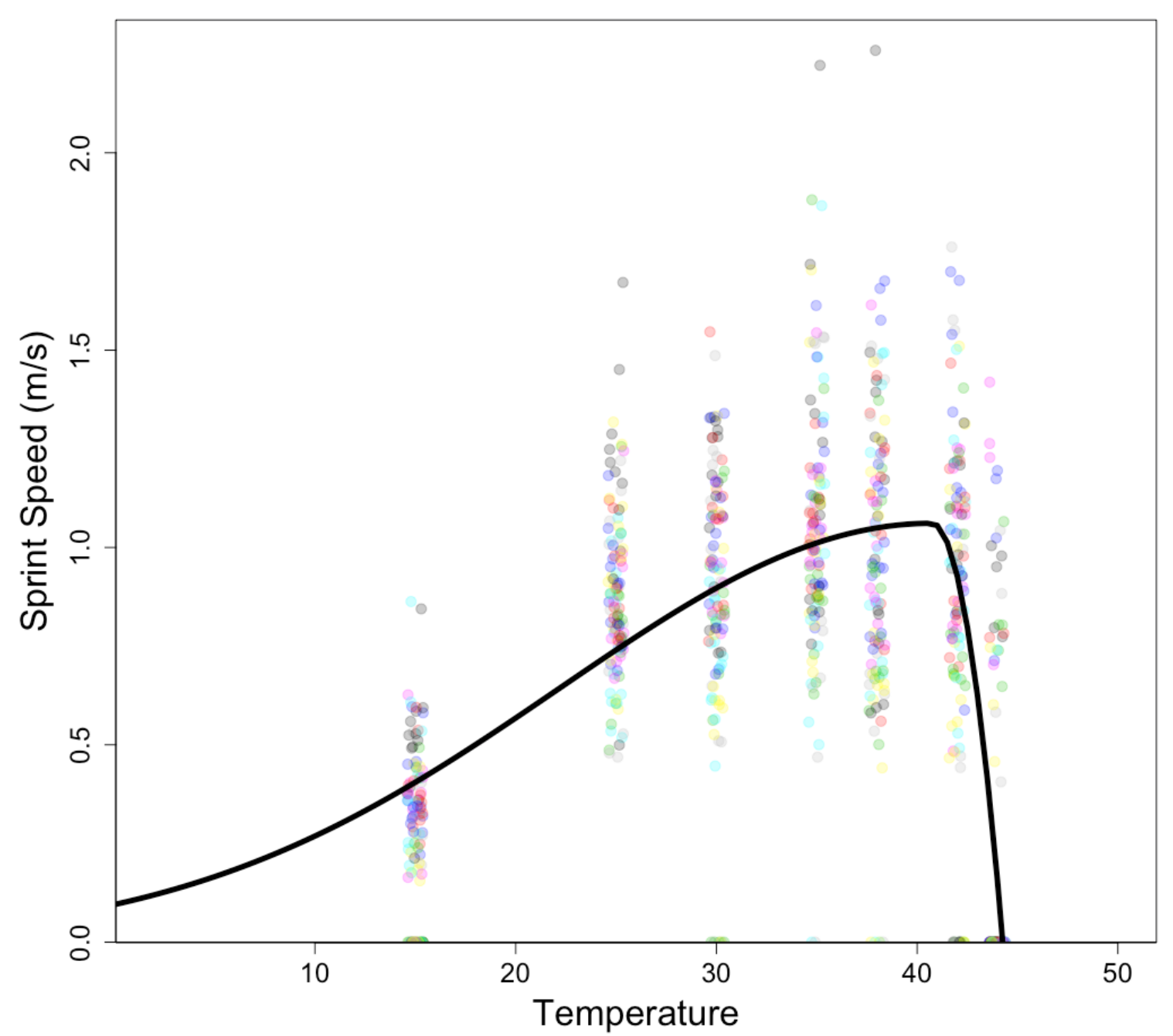

Figure S10: Estimated thermal performance of 37 male Agama atra based on three replicate measurements of sprint speed at seven temperatures. Colors indicate different individuals. Population level performance, $w$, is calculated by fitting a non-linear mixed effects model to the form: $w(T)=a\left\{\begin{array}{cc}e^{-(T-b) /(2 c)^{2}} & T \leq b \\ 1-[(T-b) /(b-d)]^{2} & T>b\end{array}\right.$ where individual $A$. atra is a random factor with respect to $\mathrm{b}$ and $\mathrm{d}$, which correspond to $C T_{\max }$ and $T_{o p t}$, respectively. This statistical relationship was fit using $\mathrm{R}$ version 3.4.1 using the nlme package.

Table S2: Parameter estimates and standard errors associated with the above model.

\begin{tabular}{lll}
\hline Parameter & Value & Standard Error \\
\hline$a$ & 1.061 & 0.019 \\
$b$ & 40.749 & 0.347 \\
$c$ & -13.119 & 0.548 \\
$d$ & 44.273 & 0.081
\end{tabular}




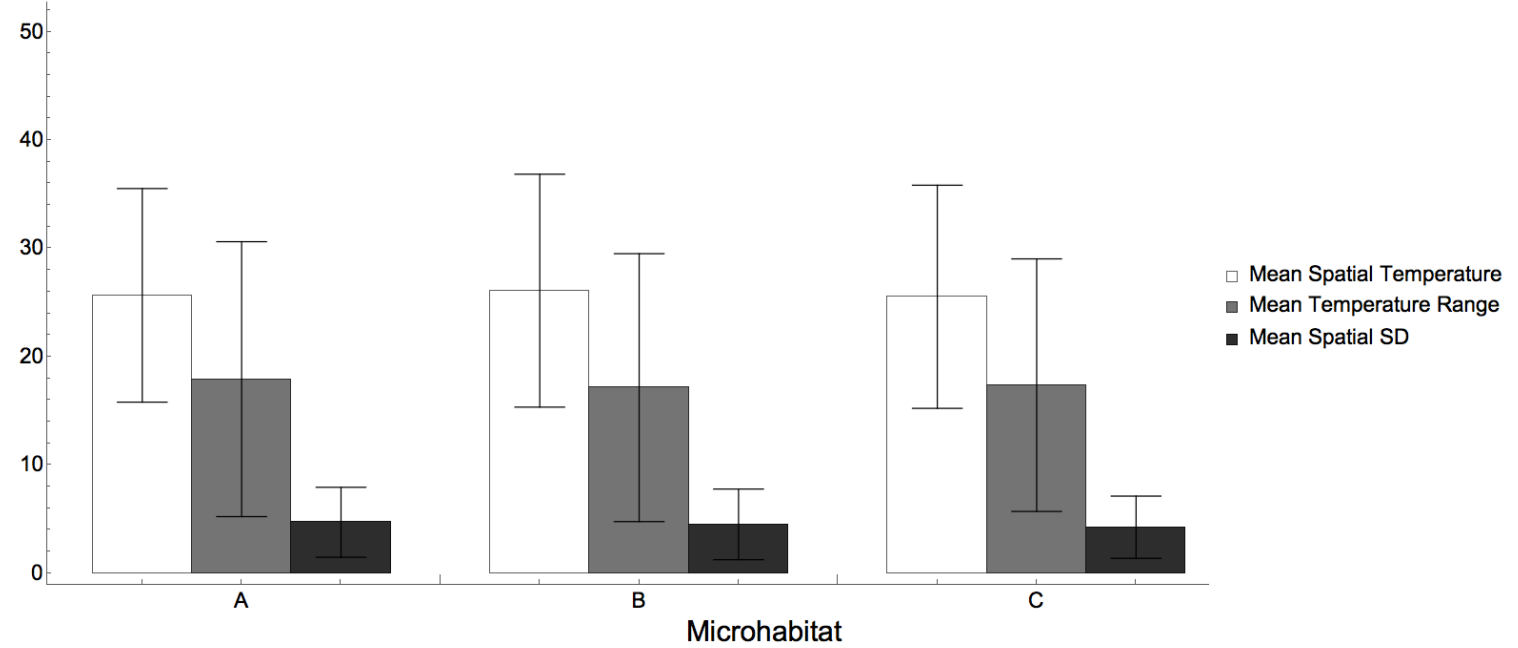

Figure S11: Mean spatial temperature (white bars; the average mean temperature each time operative temperatures are recorded), mean range of spatial temperatures (light grey bars; the range of operative temperatures recorded at each time interval), and mean spatial standard deviation (black bars; the mean standard deviation in operative temperatures) for three different $A$. atra territories (A,B,C). Territory A is analyzed in Fig. 4, Territory $\mathrm{B}$ and $\mathrm{C}$ are located nearby; the amount of variation that exists at the regional rather than microhabitat scale is additionally reported. Error bars show +/- 1 SD.
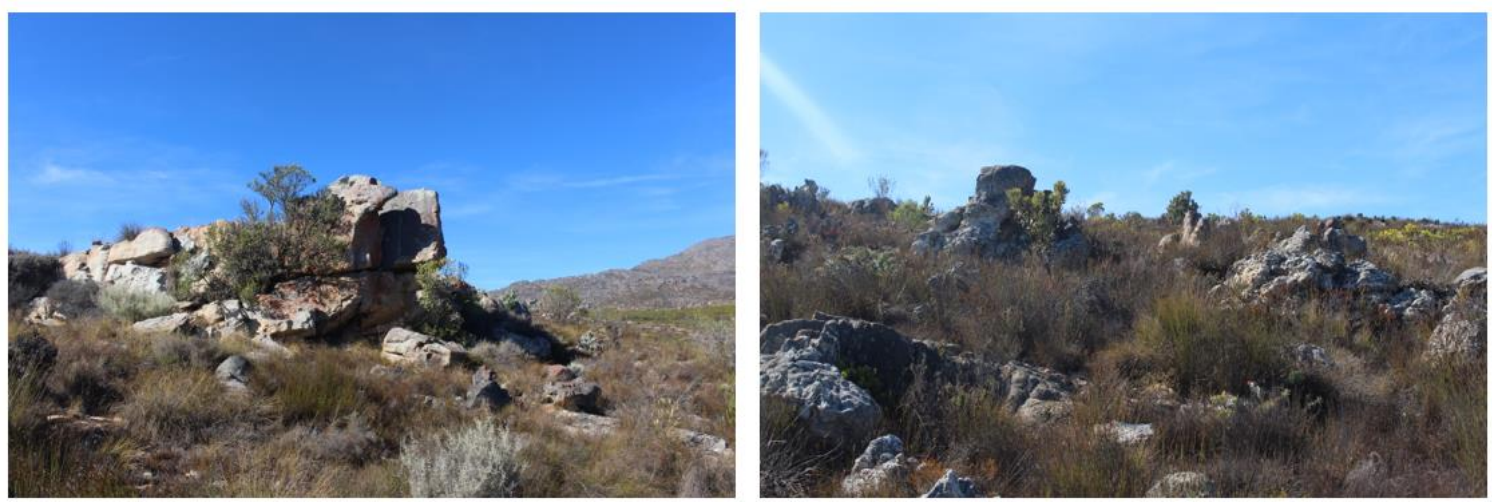

Figure S12: Photos of Agama atra territories. Photo credit Adriaan Hougaard. 

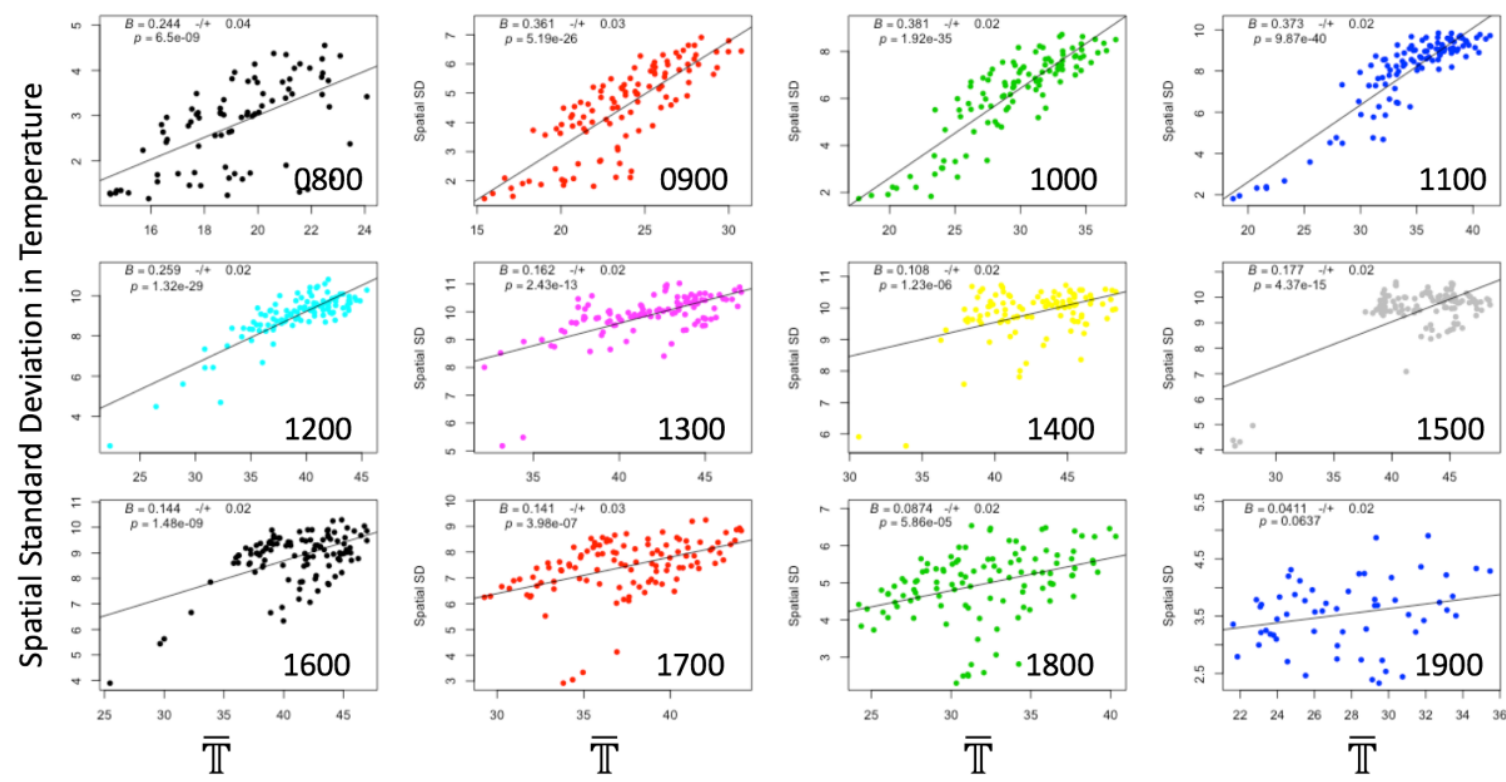

Figure S13: The spatial standard deviation of Agama atra operative temperatures increases as mean temperature increases. Each panel represents the thermal conditions during a particular hour, ranging from 0800 to 1900 . Linear slope estimates and the standard error of the slope estimates are listed in each panel. 

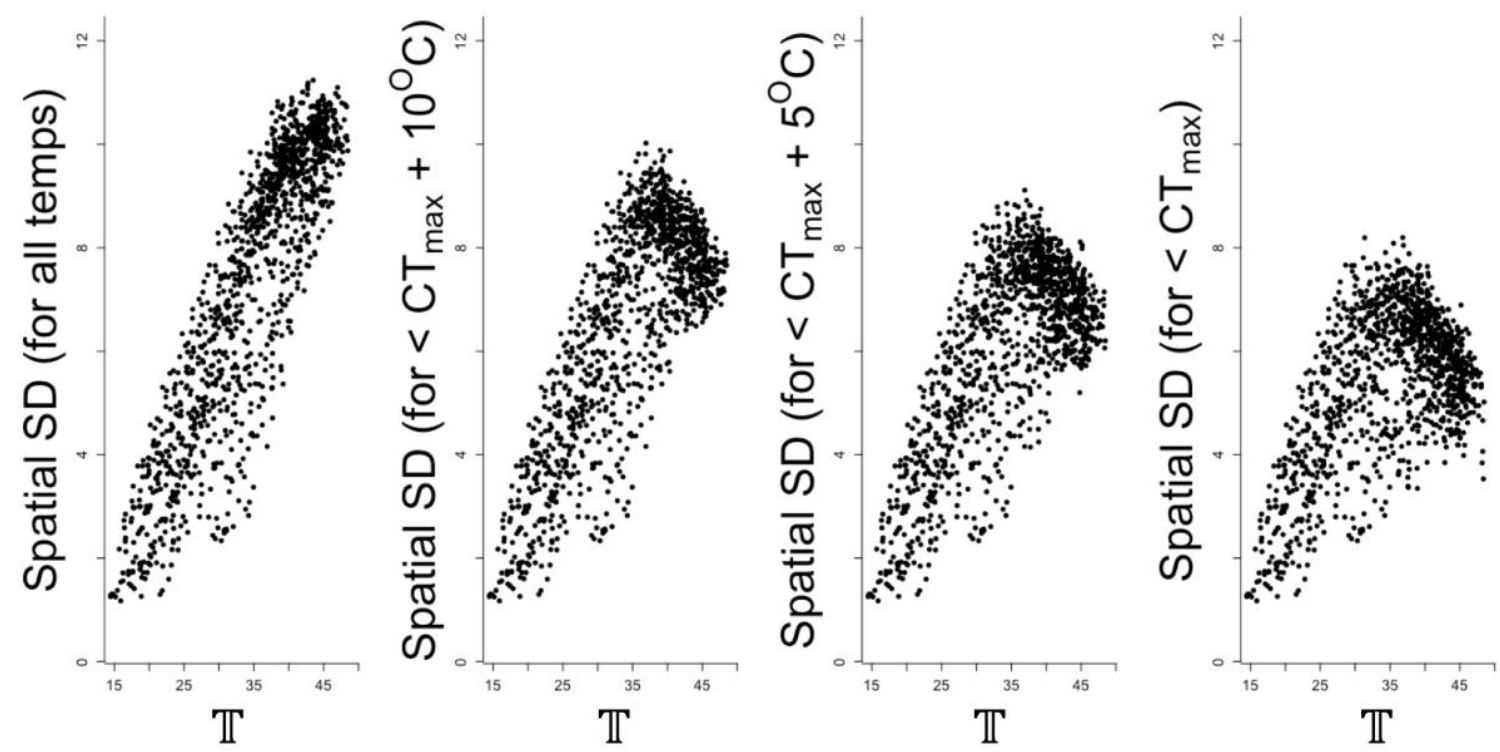

Figure S14: The relationship between spatial standard deviation in Agama atra territories, depending on the range of temperatures included. From left to right: all temperatures are considered when calculating spatial SD; only temperatures less than $54.2^{\circ} \mathrm{C}$ are considered (i.e., temps $<10^{\circ} \mathrm{C}$ above the measured $\mathrm{CT}_{\max }$ ) when calculating spatial SD; only temperatures less than $49.2^{\circ} \mathrm{C}$ are considered (i.e., $<5^{\circ} \mathrm{C}$ above the measured $\mathrm{CT}_{\max }$ ) when calculating spatial $\mathrm{SD}$; only temperatures less than $44.2^{\circ} \mathrm{C}$ are considered (i.e. temps $<\mathrm{CT}_{\max }$ ) when calculating spatial $\mathrm{SD}$.
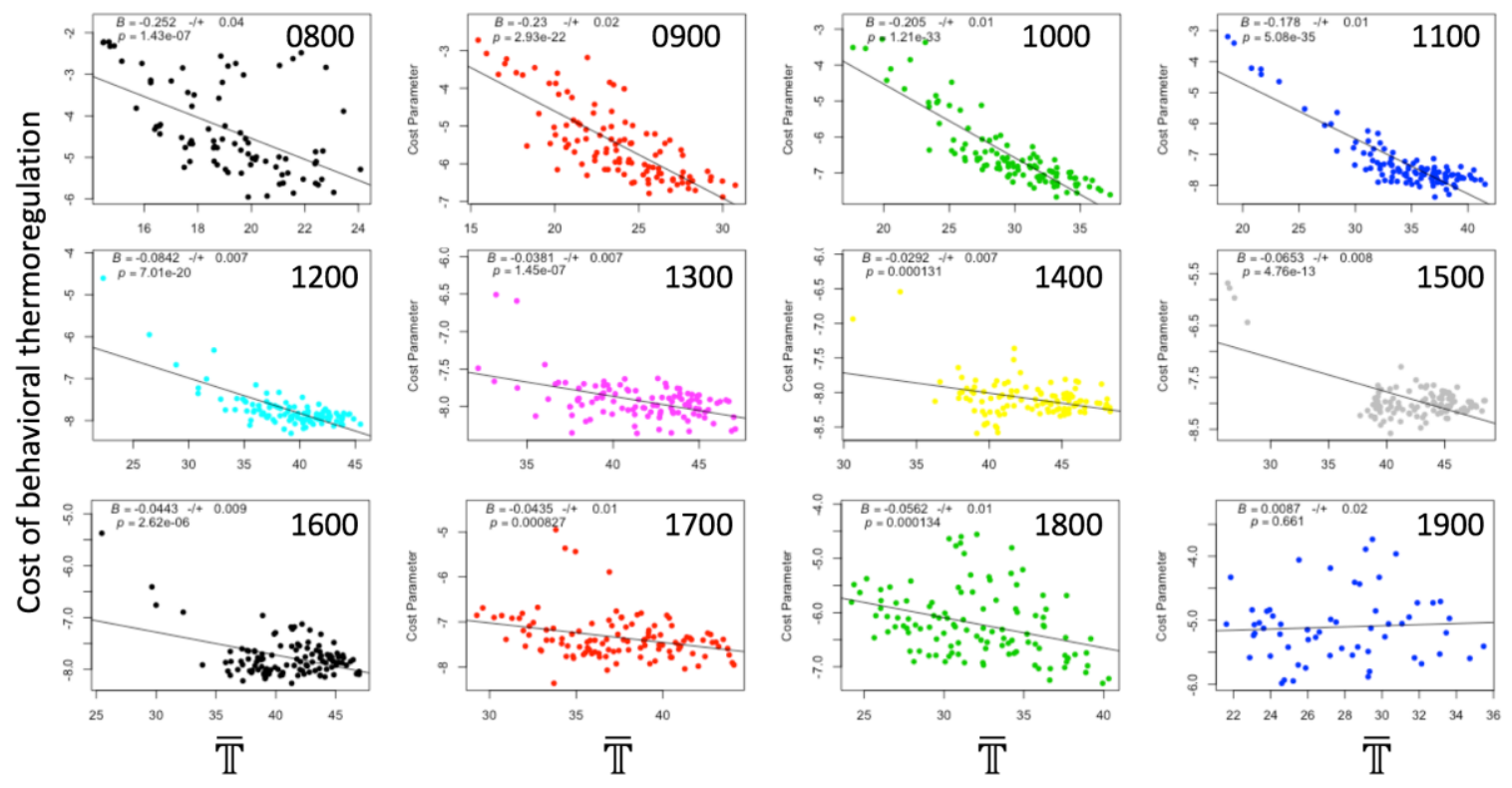

Figure S15: The cost parameters of thermoregulation in Agama atra microhabitats decreases as mean temperature increases. Each panel represents the thermal conditions during a particular hour, ranging from 0800 to 1900 . Linear slope estimates and the stand error of the slope estimates are listed on each panel. Cost parameter values correspond to $C$ in eq. 2, where lower $C$ values correspond to environments with a low cost of thermoregulation. 
Appendix 2: Supplemental methods for characterizing Agama atra territories and thermal sensitivity

We characterized the thermal heterogeneity of three individual Agama atra territories at Jonaskop ( $34^{\circ} \mathrm{S}, 1500 \mathrm{~m}$ asl), Western Cape Province, South Africa using physical models ("operative temperature models", or OTMs). Models were distributed semirandomly within each territory. OTMs were built using thin-walled, hollow copper cylinders $(80 \times 25 \times 15 \mathrm{~mm})$ and painted to approximate the skin reflectance of a typical adult $A$. atra $[3,4]$. Within each OTM, we embedded a temperature-logger (Thermochron iButton, DS1921G-F5, Sunnyvale, CA) by wrapping it in non-conductive acrylic mesh to prevent direct contact with the inside surface of the copper tube.

Thirty operative temperature models were placed within the boundaries of a lizard's territory (Fig. S12) along transects radiating at $45^{\circ}$ angles from the central basking spot (established via behavioral observations of males). To choose the location for each model, we randomized each of the following factors: (i) cardinal direction from the center of the territory; (ii) distance from the center of the territory (in $0.5 \mathrm{~m}$ intervals up to 10 m); (iii) type of substrate (rock or soil); (iv) sun exposure (sun, half-sun, shade); (v) wind exposure (exposed or sheltered); (vi) body orientation relative to sun (N, W, S or E); and (vii) slope (horizontal, $45^{\circ}$ or vertical). Sun exposure status and orientation were assigned at midday. Locations that were either in a crevice, indentation in the rock, or covered by vegetation were classified as non-wind exposed, while all others were classified as windexposed. Data were logged at 15-min intervals from 1 February to 1 March 2017. Fig. S11 shows the thermal characteristics of a typical territory (Fig. S11). 
Adult male $A$. atra lizards ( $=37$ lizards) were collected at the same site, Jonaskop, during October of 2016. Lizards were collected using the standard 'slip noose' technique [5] and transported to a laboratory at Stellenbosch University. Before measuring thermal sensitivity of performance traits, lizards were acclimated to laboratory conditions for a minimum of 5 days.

We measured the running speed of each male lizard at each of the following body temperatures: $15,25,30,35,38,42$ and $44^{\circ} \mathrm{C}$ (in random order for each individual). The choice of temperatures was based on measurements of body temperature variation of $A$. atra in the field and preliminary trials to establish their thermal window. Running speed is an ecologically relevant trait that has been linked to fitness in a number of species [6]. Lizards were heated and cooled to the target body temperature by putting them in a climate-controlled room set to that temperature for at least 1 hour prior to the start of the trial (the target temperature was verified prior to each trial using a thin thermocouple inserted in the cloaca and connected to a handheld temperature reader). Running speed was measured in an enclosed runway that was demarcated every $25 \mathrm{~cm}$ [7]. The runway was constructed out of hardboard $2.00 \mathrm{~m}$ in length, $0.15 \mathrm{~m}$ width, and $0.30 \mathrm{~m}$ height. Lizards ran on a layer of very fine sandpaper, which provided sufficient traction. We encouraged lizards to run by gently tapping their tails. Three trials were recorded at each temperature for each individual (with a minimum of 30 minutes rest between measurements at the same temperature). Trials were filmed with a high speed (60 fps) digital video camera and the maximum running speed over any $25 \mathrm{~cm}$ length of track was 
computed using frame-by-frame analysis in the motion analysis software programme Kinovea (www.kinovea.org). If the lizard could not run for at least $25 \mathrm{~cm}$ without stopping, we scored that speed as $0 \mathrm{~m} / \mathrm{s}$ [6]. All lizards were given at least two hours rest between sprint speed trials at different temperatures and we never measured the same lizard at more than two temperatures per day.

A. atra thermal performance (Fig. S10) was determined by fitting a non-linear mixed effects model with sprint speed as the response variable, averaged across three replicate measures, and individual lizard as a random factor with the form determined by Deutsch et al. [8] (Table S2).

\section{Supplementary References}

1. Alford JG, Lutterschmidt WI. 2012 Modeling energetic and theoretical costs of thermoregulatory strategy. J. Biol. Dyn. 6, 63-79.

(doi:10.1080/17513758.2011.588342)

2. Sears MW, Angilletta MJ. 2015 Costs and Benefits of Thermoregulation Revisited: Both the Heterogeneity and Spatial Structure of Temperature Drive Energetic Costs. Am. Nat. 185, E94-E102. (doi:10.1086/680008)

3. Bakken GS, Angilletta MJ. 2014 How to avoid errors when quantifying thermal environments. Funct. Ecol. 28, 96-107. (doi:10.1111/1365-2435.12149)

4. Clusella-Trullas S, van Wyk JH, Spotila JR. 2009 Thermal benefits of melanism in cordylid lizards: a theoretical and field test. Ecology 90, 2297-2312.

5. Losos JB. 2009 Lizards in an Evolutionary Tree: Ecology and Adaptive Radiation of Anoles. 1st edn. University of California Press. See http://www.jstor.org/stable/10.1525/j.ctt1pnj59.

6. Logan ML, Cox RM, Calsbeek R. 2014 Natural selection on thermal performance in a novel thermal environment. Proc. Natl. Acad. Sci. 111, 14165-14169.

(doi:10.1073/pnas.1404885111) 
7. Noble DWA, Fanson KV, Whiting MJ. 2014 Sex, androgens, and whole-organism performance in an Australian lizard. Biol. J. Linn. Soc. 111, 834-849. (doi:10.1111/bij.12252)

8. Deutsch CA, Tewksbury JJ, Huey RB, Sheldon KS, Ghalambor CK, Haak DC, Martin PR. 2008 Impacts of climate warming on terrestrial ectotherms across latitude. Proc. Natl. Acad. Sci. U. S. A. 105, 6668-6672. (doi:10.1073/pnas.0709472105) 